\title{
On the Convergence Rates of GMsFEMs for Heterogeneous Elliptic Problems without Oversampling Techniques
}

\author{
Guanglian Li*
}

September 19, 2018

\begin{abstract}
This work is concerned with the rigorous analysis on the Generalized Multiscale Finite Element Methods (GMsFEMs) for elliptic problems with high-contrast heterogeneous coefficients. GMsFEMs are popular numerical methods for solving flow problems with heterogeneous high-contrast coefficients, and it has demonstrated extremely promising numerical results for a wide range of applications. However, the mathematical justification of the efficiency of the method is still largely missing.

In this work, we analyze two types of multiscale basis functions, i.e., local spectral basis functions and basis functions of local harmonic extension type, within the GMsFEM framework. These constructions have found many applications in the past few years. We establish their optimal convergence in the energy norm under a very mild assumption that the source term belongs to some weighted $L^{2}$ space, and without the help of any oversampling technique. Furthermore, we analyze the model order reduction of the local harmonic extension basis and prove its convergence in the energy norm. These theoretical findings shed insights into the mechanism behind the efficiency of the GMsFEMs.

Keywords: multiscale methods, heterogeneous coefficient, high-contrast, elliptic problems, spectral basis function, harmonic extension basis functions, GMsFEM, proper orthogonal decomposition
\end{abstract}

\section{Introduction}

The accurate mathematical modeling of many important applications, e.g., composite materials, porous media and reservoir simulation, calls for elliptic problems with heterogeneous coefficients. In order to adequately describe the intrinsic complex properties in practical scenarios, the heterogeneous coefficients can have both multiple inseparable scales and high-contrast. Due to the disparity of scales, the classical numerical treatment becomes prohibitively expensive and even intractable for many multiscale applications. Nonetheless, motivated by the broad spectrum of practical applications, a large number of multiscale model reduction techniques, e.g., multiscale finite element methods (MsFEMs), heterogeneous multiscale methods (HMMs), variational multiscale methods, flux norm approach, generalized multiscale finite element methods (GMsFEMs) and localized orthogonal decomposition (LOD), have been proposed in the literature $[18,11,19,6,12,25,22]$ over the last few decades. They have achieved great success in the efficient and accurate simulation of heterogeneous problems. Amongst these numerical methods, the GMsFEM [12] has demonstrated extremely promising numerical results for a wide variety of problems, and thus it is becoming increasingly popular. However, the mathematical understanding of the method remains largely missing,

${ }^{*}$ Department of Mathematics, Imperial College London, London SW7 2AZ, UK. The work was partially carried out when the author was affiliated with Institut für Numerische Simulation and Hausdorff Center for Mathematics, Universität Bonn, Wegelerstraße 6, D-53115 Bonn, Germany. (lotusli0707@gmail.com, guanglian.li@imperial.ac.uk). 
despite numerous successful empirical evidences. The goal of this work is to provide a mathematical justification, by rigorously establishing the optimal convergence of the GMsFEMs in the energy norm without any restrictive assumptions or oversampling technique.

We first formulate the heterogeneous elliptic problem. Let $D \subset \mathbb{R}^{d}(d=1,2,3)$ be an open bounded Lipschitz domain with a boundary $\partial D$. Then we seek a function $u \in V:=H_{0}^{1}(D)$ such that

$$
\begin{aligned}
\mathcal{L} u:=-\nabla \cdot(\kappa \nabla u) & =f & & \text { in } D, \\
u & =0 & & \text { on } \partial D,
\end{aligned}
$$

where the force term $f \in L^{2}(D)$ and the permeability coefficient $\kappa \in L^{\infty}(D)$ with $\alpha \leq \kappa(x) \leq \beta$ almost everywhere for some lower bound $\alpha>0$ and upper bound $\beta>\alpha$. We denote by $\Lambda:=\frac{\beta}{\alpha}$ the ratio of these bounds, which reflects the contrast of the coefficient $\kappa$. Note that the existence of multiple scales in the coefficient $\kappa$ rends directly solving Problem (1.1) challenging, since resolving the problem to the finest scale would incur huge computational cost.

The goal of the GMsFEM is to efficiently capture the large-scale behavior of the solution $u$ locally without resolving all the microscale features within. To realize this desirable property, we first discretize the computational domain $D$ into a coarse mesh $\mathcal{T}^{H}$. Over $\mathcal{T}^{H}$, we define the classical multiscale basis functions $\left\{\chi_{i}\right\}_{i=1}^{N}$, with $N$ being the total number of coarse nodes. Let $\omega_{i}:=\operatorname{supp}\left(\chi_{i}\right)$ be the support of $\chi_{i}$, which is often called a local coarse neighborhood below. To accurately approximate the local solution $\left.u\right|_{\omega_{i}}$ (restricted to $\omega_{i}$ ), we construct a local approximation space. In practice, two types of local multiscale spaces are frequently employed: local spectral space $\left(V_{\text {off }}^{\mathrm{S}_{i}, \ell_{i}^{\mathrm{I}}}\right.$, of dimension $\left.\ell_{i}^{\mathrm{I}}\right)$ and local harmonic space $V_{\text {snap }}^{\mathrm{H}_{i}}$. The dimensionality of the local harmonic space $V_{\text {snap }}^{\mathrm{H}_{i}}$ is problem-dependent, and it can be extremely large when the microscale within the coefficient $\kappa$ tends to zero. Hence, a further local model reduction based on proper orthogonal decomposition (POD) in $V_{\text {snap }}^{\mathrm{H}_{i}}$ is often employed. We denote the corresponding local POD space of rank $\ell_{i}$ by $V_{\mathrm{off}}^{\mathrm{H}_{i}, \ell_{i}}$. In sum, in practice, we can have three types of local multiscale spaces at our disposal: $V_{\text {off }}^{\mathrm{S}_{i}, \ell_{i}}, V_{\text {snap }}^{\mathrm{H}_{i}}$ and $V_{\text {off }}^{\mathrm{H}_{i}, \ell_{i}}$ on $\omega_{i}$. These basis functions are then used in the standard finite element framework, e.g., continuous Galerkin formulation, for constructing a global approximate solution.

One crucial part in the local spectral basis construction is to include local spectral basis functions ( $V_{\text {off }}^{\mathrm{T}_{i}, \ell_{i}^{\mathrm{II}}}$, of dimension $\ell_{i}^{\mathrm{II}}$ ) governed by Steklov eigenvalue problems [15], which was first applied to the context of the GMsFEMs in [9], to the best of our knowledge. This was motivated by the decomposition of the local solution $\left.u\right|_{\omega_{i}}$ into the sum of three components, cf. 4.1), where the first two components can be approximated efficiently by the local spectral space $V_{\text {off }}^{\mathrm{S}_{i}, \ell_{i}^{\mathrm{I}}}$ and $V_{\text {off }}^{\mathrm{T}_{i}, \ell_{i}^{\mathrm{II}}}$, respectively, and the third component is of rank one and can be obtained by solving one local problem.

The good approximation property of these local multiscale spaces to the solution $\left.u\right|_{\omega_{i}}$ of problem (1.1) is critical to ensure the accuracy and efficiency of the GMsFEM. We shall present relevant approximation error results for the preceding three types of multiscale basis functions in Proposition 4.1, Lemma 4.3, Lemma 4.5 and Lemma4.9. It is worth pointing out that the proof of Proposition 4.1 relies crucially on the expansion of the source term $f$ in terms of the local spectral basis function in Lemma 4.2. Thus the argument differs substantially from the typical argument for such analysis that employs the oversampling argument together with a Cacciopoli type inequality [4, 13], and it is of independent interest by itself.

The proof to Lemma 4.3 is very critical. It relies essentially on the transposition method [24], which bounds the weighted $L^{2}$ error estimate in the domain by the boundary error estimate, since the latter can be obtained straightforwardly. Most importantly, the involved constant is independent of the contrast in the coefficient $\kappa$. This result is presented in Theorem A.1. 
To establish Lemmas 4.5 and 4.9 we make one mild assumption on the geometry of the coefficient, cf. Assumption 2.1, which enables the use of the weighted Friedrichs inequality in the proof. In addition, since

the local multiscale basis functions in $V_{\mathrm{off}}^{\mathrm{H}_{i}, \ell_{i}}$ are $\kappa$-harmonic and since the weighted $L^{2}\left(\omega_{i}\right)$ error estimate can be obtained directly from the POD, cf. Lemma 4.8, we employ a Cacciopoli type inequality [17] to prove Lemma 4.9. Note that our analysis does not exploit the oversampling strategy, which has played a crucial role for proving energy error estimates in all existing works [4, 13, 25, 10].

Together with the conforming Galerkin formulation and the partition of unity functions $\left\{\chi_{i}\right\}_{i=1}^{N}$ on the local domains $\left\{\omega_{i}\right\}_{i=1}^{N}$, we obtain three types of multiscale methods to solve problem (1.1), cf. (3.24)(3.26). Their energy error estimates are presented in Propositions 4.2, 4.3 and 4.4, respectively. Specifically, their convergence rates are precisely characterized by the eigenvalues $\lambda_{\ell_{i}^{\mathrm{I}}}^{\mathrm{S}_{i}}, \lambda_{\ell_{i}^{\mathrm{II}}}^{\mathrm{T}_{i}}, \lambda_{\ell_{i}}^{\mathrm{H}_{i}}$ and the coarse mesh size $H$ (see Section 4 for the definitions of the eigenvalue problems). Thus, the decay/growth behavior of these eigenvalues plays an extremely important role in determining the convergence rates, which, however, is beyond the scope of the present work. We refer readers to the works [4, 21] for results along this line.

Last, we put our contributions into the context. The local spectral estimates in the energy norm in Proposition 4.1 and Lemma 4.3 represent the state-of-art result in the sense that no restrictive assumption on the problem data is made. Furthermore, we prove the convergence without the help of the oversampling strategy in the analysis, which has played a crucial role in all existing studies [4, 14, 13, 10]. In practice, avoiding oversampling strategy allows saving computational cost, and this also corroborates well empirical observations [14]. Due to the local estimates in Proposition 4.1 and Lemma 4.3, we are able to derive a global estimate in Proposition 4.2 that is the much needed results for analyzing many multiscale methods [19, 6, 25, 22], cf. Remark 4.2. Recently Chung et al [10] proved some convergence estimates in a similar spirit to Proposition 4.1, by adapting the LOD technique [25]. Our result greatly simplifies the analysis and improves their result [10] by avoiding the oversampling. To the best of our knowledge, there is no known convergence estimate for either the local harmonic space or the local POD space, and the results presented in Propositions 4.3 and 4.4 are the first such results.

The remainder of this paper is organized as follows. We formulate the heterogeneous problem in Section 2. and describe the main idea of the GMsFEM. We present in Section 3 the construction of local multiscale spaces, harmonic extension space and discrete POD. Based upon them, we present three type of global multiscale spaces. Together with the canonical conforming Galerkin formulation, we obtain three type of numerical methods to approximate Problem (1.1) in (3.24) to 3.26). The error estimates of these multiscale methods are presented in Section 4, which represent the main contributions of this paper. Finally, we conclude the paper with concluding remarks in Section 5. We establish the regularity result of the elliptic problem with very rough boundary data in an appendix.

\section{Preliminary}

Now we present basic facts related to Problem (1.1) and briefly describe the GMsFEM (and also to fix the notation). Let the space $V:=H_{0}^{1}(D)$ be equipped with the (weighted) inner product

$$
\left\langle v_{1}, v_{2}\right\rangle_{D}=: a\left(v_{1}, v_{2}\right):=\int_{D} \kappa \nabla v_{1} \cdot \nabla v_{2} \mathrm{~d} x \quad \text { for all } v_{1}, v_{2} \in V,
$$

and the associated energy norm

$$
|v|_{H_{\kappa}^{1}(D)}^{2}:=\langle v, v\rangle_{D} \quad \text { for all } v \in V .
$$


We denote by $W:=L^{2}(D)$ equipped with the usual norm $\|\cdot\|_{L^{2}(D)}$ and inner product $(\cdot, \cdot)_{D}$.

The weak formulation for problem (1.1) is to find $u \in V$ such that

$$
a(u, v)=(f, v)_{D} \quad \text { for all } v \in V .
$$

The Lax-Milgram theorem implies the well-posedness of problem 2.1).

To discretize problem (1.1), we first introduce fine and coarse grids. Let $\mathcal{T}^{H}$ be a regular partition of the domain $D$ into finite elements (triangles, quadrilaterals, tetrahedra, etc.) with a mesh size $H$. We refer to this partition as coarse grids, and accordingly the course elements. Then each coarse element is further partitioned into a union of connected fine grid blocks. The fine-grid partition is denoted by $\mathcal{T}^{h}$ with $h$ being its mesh size. Over $\mathcal{T}^{h}$, let $V_{h}$ be the conforming piecewise linear finite element space:

$$
V_{h}:=\left\{v \in \mathcal{C}:\left.V\right|_{T} \in \mathcal{P}_{1} \text { for all } T \in \mathcal{T}^{h}\right\},
$$

where $\mathcal{P}_{1}$ denotes the space of linear polynomials. Then the fine-scale solution $u_{h} \in V_{h}$ satisfies

$$
a\left(u_{h}, v_{h}\right)=\left(f, v_{h}\right)_{D} \quad \text { for all } v_{h} \in V_{h} .
$$

The Galerkin orthogonality implies the following optimal estimate in the energy norm:

$$
\left|u-u_{h}\right|_{H_{\kappa}^{1}(D)} \leq \min _{v_{h} \in V_{h}}\left|u-v_{h}\right|_{H_{\kappa}^{1}(D)}
$$

The fine-scale solution $u_{h}$ will serve as a reference solution in multiscale methods. Note that due to the presence of multiple scales in the coefficient $\kappa$, the fine-scale mesh size $h$ should be commensurate with the smallest scale and thus it can be very small in order to obtain an accurate solution. This necessarily involves huge computational complexity, and more efficient methods are in great demand.

In this work, we are concerned with flow problems with high-contrast heterogeneous coefficients, which involve multiscale permeability fields, e.g., permeability fields with vugs and faults, and furthermore, can be parameter-dependent, e.g., viscosity. Under such scenario, the computation of the fine-scale solution $u_{h}$ is vulnerable to high computational complexity, and one has to resort to multiscale methods. The GMsFEM has been extremely successful for solving multiscale flow problems, which we briefly recap below.

The GMsFEM aims at solving Problem (1.1) on the coarse mesh $\mathcal{T}^{H}$ cheaply, which, meanwhile, maintains a certain accuracy compared to the fine-scale solution $u_{h}$. To describe the GMsFEM, we need a few notation. The vertices of $\mathcal{T}^{H}$ are denoted by $\left\{O_{i}\right\}_{i=1}^{N}$, with $N$ being the total number of coarse nodes. The coarse neighborhood associated with the node $O_{i}$ is denoted by

$$
\omega_{i}:=\bigcup\left\{K_{j} \in \mathcal{T}^{H}: \quad O_{i} \in \bar{K}_{j}\right\} .
$$

The overlap constant $C_{\mathrm{ov}}$ is defined by

$$
C_{\mathrm{ov}}:=\max _{K \in \mathcal{T}^{H}} \#\left\{O_{i}: K \subset \omega_{i} \text { for } i=1,2, \cdots, N\right\} .
$$

We refer to Figure 1 for an illustration of neighborhoods and elements subordinated to the coarse discretization $\mathcal{T}^{H}$. Throughout, we use $\omega_{i}$ to denote a coarse neighborhood.

Next, we outline the GMsFEM with a continuous Galerkin (CG) formulation; see Section 3 for details. We denote by $\omega_{i}$ the support of the multiscale basis functions. These basis functions are denoted by $\psi_{k}^{\omega_{i}}$ for $k=1, \cdots, \ell_{i}$ for some $\ell_{i} \in \mathbb{N}_{+}$, which is the number of local basis functions associated with $\omega_{i}$. Throughout, the superscript $i$ denotes the $i$-th coarse node or coarse neighborhood $\omega_{i}$. Generally, the GMsFEM 


\section{$\mathcal{T}^{H}$ (Coarse Grid)}

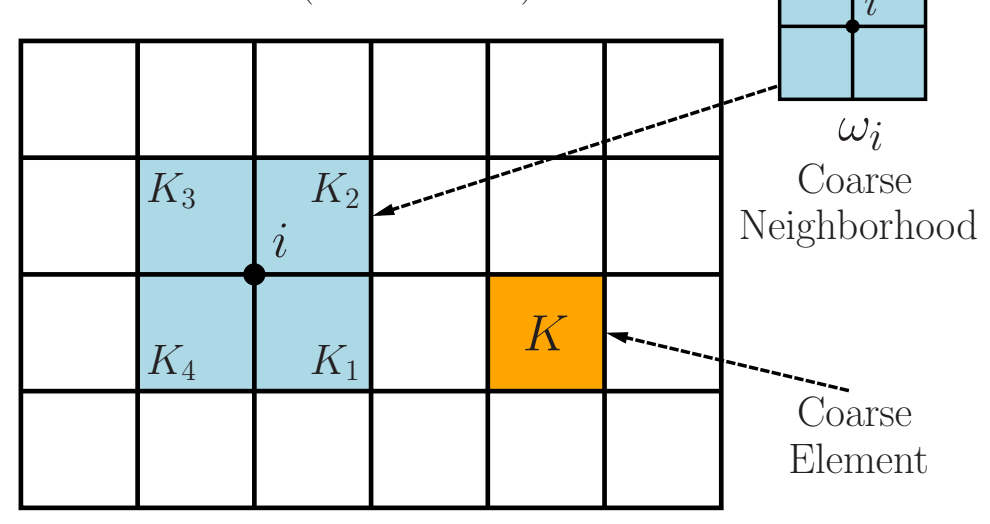

Figure 1: Illustration of a coarse neighborhood and coarse element with an overlapping constant $C_{\mathrm{ov}}=4$.

utilizes multiple basis functions per coarse neighborhood $\omega_{i}$, and the index $k$ represents the numbering of these basis functions. In turn, the CG multiscale solution $u_{\mathrm{ms}}$ is sought as $u_{\mathrm{ms}}(x)=\sum_{i, k} c_{k}^{i} \psi_{k}^{\omega_{i}}(x)$. Once the basis functions $\psi_{k}^{\omega_{i}}$ are identified, the CG global coupling is given through the variational form

$$
a\left(u_{\mathrm{ms}}, v\right)=(f, v), \quad \text { for all } v \in V_{\text {off }},
$$

where $V_{\text {off }}$ denotes the finite element space spanned by these basis functions.

We conclude the section with the following assumption on $\Omega$ and $\kappa$.

Assumption 2.1 (Structure of $D$ and $\kappa$ ). Let $D$ be a domain with a $C^{1, \alpha}(0<\alpha<1)$ boundary $\partial D$, and $\left\{D_{i}\right\}_{i=1}^{m} \subset D$ be $m$ pairwise disjoint strictly convex open subsets, each with a $C^{1, \alpha}$ boundary $\Gamma_{i}:=\partial D_{i}$, and denote $D_{0}=D \backslash \overline{\cup_{i=1}^{m} D_{i}}$. Let the permeability coefficient $\kappa$ be piecewise regular function defined by

$$
\kappa= \begin{cases}\eta_{i}(x) & \text { in } D_{i}, \\ 1 & \text { in } D_{0} .\end{cases}
$$

Here $\eta_{i} \in C^{\mu}\left(\bar{D}_{i}\right)$ with $\mu \in(0,1)$ for $i=1, \cdots, m$. Denote $\eta_{\min }:=\min _{i}\left\{\eta_{i}\right\} \geq 1$ and $\eta_{\max }:=\max _{i}\left\{\eta_{i}\right\}$.

Under Assumption 2.1, the coefficient $\kappa$ is $\Gamma$-quasi-monotone on each coarse neighborhood $\omega_{i}$ and the global domain $D$ (see [27, Definition 2.6] for the precise definition) with either $\Gamma:=\partial \omega_{i}$ or $\Gamma:=\partial D$. Then the following weighted Friedrichs inequality [27, Theorem 2.7] holds.

Theorem 2.1 (Weighted Friedrichs inequality). Let diam $(D)$ be the diameter of the bounded domain $D$ and $\omega_{i} \subset$ D. Define

$$
\begin{aligned}
\mathrm{C}_{\text {poin }}\left(\omega_{i}\right) & :=H^{-2} \max _{w \in H_{0}^{1}\left(\omega_{i}\right)} \frac{\int_{\omega_{i}} \kappa w^{2} \mathrm{~d} x}{\int_{\omega_{i}} \kappa|\nabla w|^{2} \mathrm{~d} x}, \\
\mathrm{C}_{\text {poin }}(D) & :=\operatorname{diam}(D)^{-2} \max _{w \in H_{0}^{1}(D)} \frac{\int_{D} \kappa w^{2} \mathrm{~d} x}{\int_{D} \kappa|\nabla w|^{2} \mathrm{~d} x} .
\end{aligned}
$$

Then the positive constants $\mathrm{C}_{\text {poin }}\left(\omega_{i}\right)$ and $\mathrm{C}_{\text {poin }}(D)$ are independent of the contrast of $\kappa$.

Remark 2.1. Below we only require that the constants $\mathrm{C}_{\text {poin }}\left(\omega_{i}\right)$ and $\mathrm{C}_{\text {poin }}(D)$ be independent of the contrast in $\kappa$. Assumption 2.1] is one sufficient condition to ensure this, and it can be relaxed [27]. 


\section{CG-based GMsFEM for high-contrast flow problems}

In this section, we present the local spectral basis functions, local harmonic extension basis functions and POD, and the global weak formulation based on these local multiscale basis functions.

\subsection{Local multiscale basis functions}

First we present two principled approaches for constructing local multiscale functions: local spectral bases and local harmonic extension bases, which represent the two main approaches within the GMsFEM framework. The constructions are carried out on each coarse neighborhood $\omega_{i}$ with $i=1,2, \cdots, N$, and can be carried out in parallel, if desired. Since the dimensionality of the local harmonic extension bases is problemdependent and inversely proportional to the smallest scale in $\kappa$, in practice, we often perform an "optimal" local model order reduction based on POD to further reduce the complexity at the online stage.

Before presenting the constructions, we first introduce some useful function spaces, which will play an important role in the analysis below. Let $L_{\widetilde{\kappa}}^{2}\left(\omega_{i}\right)$ and $H_{\kappa}^{1}\left(\omega_{i}\right)$ be Hilbert spaces with their inner products and norms defined respectively by

$$
\begin{aligned}
\left(w_{1}, w_{2}\right)_{i} & :=\int_{\omega_{i}} \widetilde{\kappa} w_{1} \cdot w_{2} \mathrm{~d} x & \left\|w_{1}\right\|_{L_{\widetilde{\kappa}}^{2}\left(\omega_{i}\right)}^{2}:=\left(w_{1}, w_{1}\right)_{i} & \text { for } w_{1}, w_{2} \in L_{\widetilde{\kappa}}^{2}\left(\omega_{i}\right), \\
\left\langle v_{1}, v_{2}\right\rangle_{i} & :=\int_{\omega_{i}} \kappa \nabla v_{1} \cdot \nabla v_{2} \mathrm{~d} x & \left\|v_{1}\right\|_{H_{\kappa}^{1}\left(\omega_{i}\right)}^{2}:=\left(v_{1}, v_{2}\right)_{i}+\left\langle v_{1}, v_{1}\right\rangle_{i} & \text { for } v_{1}, v_{2} \in H_{\kappa}^{1}\left(\omega_{i}\right) .
\end{aligned}
$$

Next we define two subspaces $W_{i} \subset L_{\widetilde{\kappa}}^{2}\left(\omega_{i}\right)$ and $V_{i} \subset H_{\kappa}^{1}\left(\omega_{i}\right)$ of codimension one by

$$
W_{i}:=\left\{v \in L_{\widetilde{\kappa}}^{2}\left(\omega_{i}\right): \int_{\omega_{i}} \widetilde{\kappa} v \mathrm{~d} x=0\right\} \quad \text { and } \quad V_{i}:=\left\{v \in H_{\kappa}^{1}\left(\omega_{i}\right): \int_{\omega_{i}} \widetilde{\kappa} v \mathrm{~d} x=0\right\} .
$$

Furthermore, we introduce the following weighted Sobolev spaces:

$$
\begin{aligned}
L_{\kappa^{-1}}^{2}\left(\omega_{i}\right) & :=\left\{w:\|w\|_{L_{\kappa^{-1}\left(\omega_{i}\right)}^{2}}^{2}:=\int_{\omega_{i}} \kappa^{-1} w^{2} \mathrm{~d} x<\infty\right\}, \\
H_{\kappa, 0}^{1}\left(\omega_{i}\right) & :=\left\{w:\left.w\right|_{\partial \omega_{i}}=0 \text { s.t. }|w|_{H_{\kappa}^{1}\left(\omega_{i}\right)}^{2}:=\int_{\omega_{i}} \kappa|\nabla w|^{2} \mathrm{~d} x<\infty\right\} .
\end{aligned}
$$

Similarly, we define the following weighted Sobolev spaces with their associated norms: $\left(L_{\widetilde{\kappa}^{-1}}^{2}\left(\omega_{i}\right), \|\right.$.

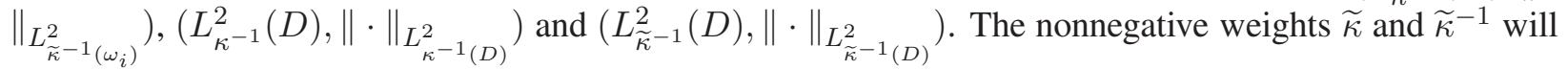
be defined in (3.3) and (3.4) below, respectively.

Throughout, the superscripts $\mathrm{S}_{i}, \mathrm{~T}_{i}$ and $\mathrm{H}_{i}$ are associated to the local spectral spaces and local harmonic space on $\omega_{i}$, respectively. Below we describe the construction of local multiscale basis functions on $\omega_{i}$.

\section{Local spectral bases I}

To define the local spectral bases on $\omega_{i}$, we first introduce a local elliptic operator $\mathcal{L}_{i}$ on $\omega_{i}$ by

$$
\left\{\begin{array}{l}
\mathcal{L}_{i} v:=-\nabla \cdot(\kappa \nabla v) \quad \text { in } \omega_{i} \\
\kappa \frac{\partial v}{\partial n}=0 \quad \text { on } \partial \omega_{i} .
\end{array}\right.
$$


The Lax-Milgram theorem implies the well-posedness of the operator $\mathcal{L}_{i}: V_{i} \rightarrow V_{i}^{*}$, the dual space $V_{i}^{*}$ of $V_{i}$. Then the spectral problem can be formulated in terms of $\mathcal{L}_{i}$, i.e., to seek $\left(\lambda_{j}^{\mathrm{S}_{i}}, v_{j}^{\mathrm{S}_{i}}\right) \in \mathbb{R} \times V_{i}$ such that

$$
\begin{aligned}
\mathcal{L}_{i} v_{j}^{\mathrm{S}_{i}} & =\widetilde{\kappa} \lambda_{j}^{\mathrm{S}_{i}} v_{j}^{\mathrm{S}_{i}} & & \text { in } \omega_{i}, \\
\kappa \frac{\partial}{\partial n} v_{j}^{\mathrm{S}_{i}} & =0 & & \text { on } \partial \omega_{i},
\end{aligned}
$$

where the parameter $\widetilde{\kappa}$ is defined by

$$
\widetilde{\kappa}=H^{2} \kappa \sum_{i=1}^{N}\left|\nabla \chi_{i}\right|^{2},
$$

with the multiscale function $\chi_{i}$ to be defined in (3.20) below. Note that the use of $\widetilde{\kappa}$ in the local spectral problem (3.2) instead of $\kappa$ is due to numerical consideration [14]. Furthermore, let $\widetilde{\kappa}^{-1}$ be defined by

$$
\widetilde{\kappa}^{-1}(x)= \begin{cases}\widetilde{\kappa}^{-1}, & \text { when } \widetilde{\kappa}(x) \neq 0 \\ 1, & \text { otherwise }\end{cases}
$$

Remark 3.1. Generally, one cannot preclude the existence of critical points from the multiscale basis functions $\chi_{i}[3$ [2]. In the two-dimensional case, it was proved that there are at most a finite number of isolated critical points. To simplify our presentation, we will assume $|D \cap\{\widetilde{\kappa}=0\}|=0$.

The next result gives the eigenvalue behavior of the local spectral problem (3.2).

Theorem 3.1. Let $\left\{\left(\lambda_{j}^{\mathrm{S}_{i}}, v_{j}^{\mathrm{S}_{i}}\right)\right\}_{j=1}^{\infty}$ be the eigenvalues and the corresponding normalized eigenfunctions in $W_{i}$ to the spectral problem (3.2) listed according to their algebraic multiplicities and the eigenvalues are ordered nondecreasingly. There holds

$$
\lambda_{j}^{\mathrm{S}_{i}} \rightarrow \infty \quad \text { as } j \rightarrow \infty
$$

To prove Theorem 3.1, we need a few notation. Let $\mathcal{S}_{i}:=\mathcal{L}_{i}^{-1}: V_{i}^{*} \rightarrow V_{i}$ be the inverse of the elliptic operator $\mathcal{L}_{i}$. Denote $T: W_{i} \rightarrow L_{\widetilde{\kappa}^{-1}}^{2}\left(\omega_{i}\right)$ to be the multiplication operator defined by

$$
T v:=\widetilde{\kappa} v \quad \text { for all } \quad v \in W_{i} .
$$

One can show by definition directly that $T$ is a bounded operator with unit norm. Moreover, there holds

$$
\int_{\omega_{i}} T v \mathrm{~d} x=0 \quad \text { for all } v \in W_{i}
$$

Thus the range of $T, \mathcal{R}(T)$, is a subspace in $L_{\widetilde{\kappa}^{-1}}^{2}\left(\omega_{i}\right)$ with codimension one, and we have

$$
\mathcal{R}(T) \hookrightarrow V_{i}^{*} .
$$

For the proof of Theorem 3.1, we need the following compact embedding result.

Lemma 3.1. $V_{i}$ is compactly embedded into $W_{i}$, i.e., $V_{i} \hookrightarrow \hookrightarrow W_{i}$. 
Proof. By Remark 3.3 , the uniform boundedness of $\kappa$, the definition of $\widetilde{\kappa}$ and the overlapping condition (2.5), we obtain the boundedness of $\tilde{\kappa}$, i.e.,

$$
\|\widetilde{\kappa}\|_{L^{\infty}(D)} \leq C_{\mathrm{ov}}\left(H C_{0}\right)^{2} \kappa \leq C_{\mathrm{ov}}\left(H C_{0}\right)^{2} \beta .
$$

Hence, there holds the following embedding inequalities:

$$
L_{\widetilde{\kappa}^{-1}}^{2}\left(\omega_{i}\right) \hookrightarrow L^{2}\left(\omega_{i}\right) \hookrightarrow L_{\widetilde{\kappa}}^{2}\left(\omega_{i}\right) .
$$

This, the classical Sobolev embedding [1] and boundedness of $\kappa$ imply the compactness of the embedding $V_{i} \hookrightarrow \hookrightarrow L^{2}\left(\omega_{i}\right)$ and thus, we finally arrive at $V_{i} \hookrightarrow \hookrightarrow W_{i}$. This completes the proof.

Proof of Theorem 3.1 By [3.7), the multiplication operator $T: W_{i} \rightarrow V_{i}^{*}$ is bounded. Similarly, the operator $\mathcal{S}_{i}: V_{i}^{*} \rightarrow W_{i}$ is compact, in view of Lemma3.1. Let $\widetilde{\mathcal{S}}_{i}:=\mathcal{S}_{i} T$. Then the operator $\widetilde{\mathcal{S}}_{i}: W_{i} \rightarrow W_{i}$ is nonnegative and compact. Now we claim that $\widetilde{\mathcal{S}}_{i}$ is self-adjoint on $W_{i}$. Indeed, for all $v, w \in W_{i}$, we have

$$
\begin{aligned}
\left(\widetilde{\mathcal{S}}_{i} v, w\right)_{i} & =\left(\mathcal{S}_{i} T v, w\right)_{i}=\int_{\omega_{i}} \widetilde{\kappa} \mathcal{L}_{i}^{-1}(\widetilde{\kappa} v) w \mathrm{~d} x \\
& =\int_{\omega_{i}} \mathcal{L}_{i}^{-1}(\widetilde{\kappa} v)(\widetilde{\kappa} w) \mathrm{d} x \\
& =\left(v,\left(\mathcal{S}_{i} T\right) w\right)_{i}=\left(v, \widetilde{\mathcal{S}}_{i} w\right)_{i},
\end{aligned}
$$

where we have used the weak formulation for (3.1) to deduce $\int_{\omega_{i}} \mathcal{L}_{i}^{-1}(\widetilde{\kappa} v)(\widetilde{\kappa} w) \mathrm{d} x=\int_{\omega_{i}}(\widetilde{\kappa} v) \mathcal{L}_{i}^{-1}(\widetilde{\kappa} w) \mathrm{d} x$. By the standard spectral theory for compact operators [28], it has at most countably many discrete eigenvalues, with zero being the only accumulation point, and each nonzero eigenvalue has only finite multiplicity. Noting that $\left\{\left(\left(\lambda_{j}^{\mathrm{S}_{i}}\right)^{-1}, v_{j}^{\mathrm{S}_{i}}\right)\right\}_{j=1}^{\infty}$ are the eigenpairs of $\widetilde{\mathcal{S}}_{i}$ completes the proof.

Furthermore, by the construction, the eigenfunctions $\left\{v_{j}^{\mathrm{S}_{i}}\right\}_{j=1}^{\infty}$ form a complete orthonormal bases $(\mathrm{CONB})$ in $W_{i}$, and $\left\{\sqrt{\lambda_{j}^{\mathrm{S}_{i}}+1} v_{j}^{\mathrm{S}_{i}}\right\}_{j=1}^{\infty}$ form a CONB in $V_{i}$. Further, we have $L_{\widetilde{\kappa}}^{2}\left(\omega_{i}\right)=W_{i} \oplus\{1\}$. Hence, $\left\{v_{j}^{\mathrm{S}_{i}}\right\}_{j=1}^{\infty} \oplus\{1\}$ is a complete orthogonal bases in $L_{\widetilde{\kappa}}^{2}\left(\omega_{i}\right)$ [Chapters 4 and 5][20]].

Lemma 3.2. The series $\left\{\widetilde{\kappa} v_{j}^{\mathrm{S}_{i}}\right\}_{j=1}^{\infty} \oplus\{\widetilde{\kappa}\}$ forms a complete orthogonal bases in $L_{\widetilde{\kappa}^{-1}}^{2}\left(\omega_{i}\right)$.

Proof. First, we show that $\left\{\widetilde{\kappa} v_{j}^{\mathrm{S}_{i}}\right\}_{j=1}^{\infty} \oplus\{\widetilde{\kappa}\}$ are orthogonal in $L_{\widetilde{\kappa}^{-1}}^{2}\left(\omega_{i}\right)$. Indeed, by definition, we deduce that for all $j \in \mathbb{N}_{+}$

$$
\int_{\omega_{i}} \widetilde{\kappa}^{-1} \widetilde{\kappa} \cdot \widetilde{\kappa} v_{j}^{\mathrm{S}_{i}} \mathrm{~d} x=\int_{\omega_{i}} \widetilde{\kappa} v_{j}^{\mathrm{S}_{i}} \mathrm{~d} x=\left(v_{j}^{\mathrm{S}_{i}}, 1\right)_{i}=0 .
$$

Meanwhile, for all $j, k \in \mathbb{N}_{+}$, there holds

$$
\int_{\omega_{i}} \widetilde{\kappa}^{-1} \widetilde{\kappa} v_{k}^{\mathrm{S}_{i}} \cdot \widetilde{\kappa} v_{j}^{\mathrm{S}_{i}} \mathrm{~d} x=\int_{\omega_{i}} \widetilde{\kappa} v_{j}^{\mathrm{S}_{i}} \cdot v_{k}^{\mathrm{S}_{i}} \mathrm{~d} x=\left(v_{j}^{\mathrm{S}_{i}}, v_{k}^{\mathrm{S}_{i}}\right)_{i}=\delta_{j, k} .
$$

Next we show that $\left\{\widetilde{\kappa} v_{j}^{\mathrm{S}_{i}}\right\}_{j=1}^{\infty} \oplus\{\widetilde{\kappa}\}$ are complete in $L_{\widetilde{\kappa}^{-1}}^{2}\left(\omega_{i}\right)$. Actually, for any $v \in L_{\widetilde{\kappa}^{-1}}^{2}\left(\omega_{i}\right)$ such that

$$
\int_{\omega_{i}} \widetilde{\kappa}^{-1} v \cdot \widetilde{\kappa} \mathrm{d} x=0 \quad \text { and } \quad \forall j \in \mathbb{N}_{+}: \int_{\omega_{i}} \widetilde{\kappa}^{-1} v \cdot \widetilde{\kappa} v_{j}^{\mathrm{S}_{i}} \mathrm{~d} x=0
$$

\footnotetext{
${ }^{1}$ We thank Richard S. Laugesen (University of Illinois, Urbana-Champaign) for clarifying the convergence in $H_{\kappa}^{1}\left(\omega_{i}\right)$.
} 
we deduce directly from definition that

$$
\int_{\omega_{i}} \widetilde{\kappa}\left(\widetilde{\kappa}^{-1} v\right)^{2} \mathrm{~d} x=\int_{\omega_{i} \cap\{\widetilde{\kappa} \neq 0\}} \widetilde{\kappa}^{-1} v^{2} \mathrm{~d} x<\infty .
$$

This implies that $\widetilde{\kappa}^{-1} v \in L_{\widetilde{\kappa}}^{2}\left(\omega_{i}\right)$. Furthermore, (3.9) indicates that $\widetilde{\kappa}^{-1} v$ is orthogonal to a set of complete orthogonal basis functions $\left\{v_{j}^{\mathrm{S}_{i}}\right\}_{j=1}^{\infty} \oplus\{1\}$ in $L_{\widetilde{\kappa}}^{2}\left(\omega_{i}\right)$. Therefore, $v=0$, which completes the proof.

Remark 3.2. Since $L_{\widetilde{\kappa}^{-1}}^{2}\left(\omega_{i}\right)$ is a Hilbert space, we can identify its dual with itself, and there exists an isometry between $L_{\widetilde{\kappa}}^{2}\left(\omega_{i}\right)$ and $L_{\widetilde{\kappa}^{-1}}^{2}\left(\omega_{i}\right)$, e.g., the operator $T$ in (3.6). We identify $L_{\widetilde{\kappa}}^{2}\left(\omega_{i}\right)$ as the dual of $L_{\widetilde{\kappa}^{-1}}^{2}\left(\omega_{i}\right)$.

Now we define the local spectral basis functions on $\omega_{i}$ for all $i=1, \cdots, N$. Let $\ell_{i}^{\mathrm{I}} \in \mathbb{N}_{+}$be a prespecified number, denoting the number of local basis functions associated with $\omega_{i}$. We take the eigenfunctions corresponding to the first $\left(\ell_{i}^{\mathrm{I}}-1\right)$ smallest eigenvalues for problem (3.2) in addition to the kernel of the elliptic operator $\mathcal{L}_{i}$, namely, $\{1\}$, to construct the local spectral offline space:

$$
V_{\text {off }}^{\mathrm{S}_{i}, \ell_{i}^{\mathrm{I}}}=\operatorname{span}\left\{v_{j}^{\mathrm{S}_{i}}: \quad 1 \leq j<\ell_{i}^{\mathrm{I}}\right\} \oplus\{1\} .
$$

Then $\operatorname{dim}\left(V_{\text {off }}^{\mathrm{S}_{i}, \ell_{i}^{\mathrm{I}}}\right)=\ell_{i}^{\mathrm{I}}$. The choice of the truncation number $\ell_{i}^{\mathrm{I}} \in \mathbb{N}_{+}$has to be determined by the eigenvalue decay rate or the presence of spectral gap. The space $V_{\text {off }}^{\mathrm{S}_{i}, \ell_{i}^{\mathrm{I}}}$ allows defining a finite-rank projection operator $\mathcal{P}^{\mathrm{S}_{i}, \ell_{i}^{\mathrm{I}}}: L_{\widetilde{\kappa}}^{2}\left(\omega_{i}\right) \rightarrow V_{\text {off }}^{\mathrm{S}_{i}, \ell_{i}^{\mathrm{I}}}$ by (with the constant $\left.c_{0}=\left(\int_{\omega_{i}} \widetilde{\kappa} \mathrm{d} x\right)^{-1}\right)$ :

$$
\mathcal{P}^{\mathrm{S}_{i}, \ell_{i}^{\mathrm{I}}} v=c_{0}(v, 1)_{i}+\sum_{j=1}^{\ell_{i}^{\mathrm{I}}-1}\left(v, v_{j}^{\mathrm{S}_{i}}\right)_{i} v_{j}^{\mathrm{S}_{i}} \quad \text { for all } v \in L_{\tilde{\kappa}}^{2}\left(\omega_{i}\right) .
$$

The operator $\mathcal{P}^{\mathrm{S}_{i}, \ell_{i}^{\mathrm{I}}}$ will play a role in the convergence analysis.

\section{Local Steklov eigenvalue problem II}

The local Steklov eigenvalue problem can be formulated as to seeking $\left(\lambda_{j}^{\mathrm{T}_{i}}, v_{j}^{\mathrm{T}_{i}}\right) \in \mathbb{R} \times H_{\kappa}^{1}\left(\omega_{i}\right)$ such that

$$
\begin{aligned}
-\nabla \cdot\left(\kappa \nabla v_{j}^{\mathrm{T}_{i}}\right) & =0 & & \text { in } \omega_{i}, \\
\kappa \frac{\partial}{\partial n} v_{j}^{\mathrm{T}_{i}} & =\lambda_{j}^{\mathrm{T}_{i}} v_{j}^{\mathrm{T}_{i}} & & \text { on } \partial \omega_{i} .
\end{aligned}
$$

It is well known that the spectrals of the Steklov eigenvalue problem blow up [15]:

Theorem 3.2. Let $\left\{\left(\lambda_{j}^{\mathrm{T}_{i}}, v_{j}^{\mathrm{T}_{i}}\right)\right\}_{j=1}^{\infty}$ be the eigenvalues and the corresponding normalized eigenfunctions in $L^{2}\left(\partial \omega_{i}\right)$ to the spectral problem (3.11) listed according to their algebraic multiplicities and the eigenvalues are ordered nondecreasingly. There holds

$$
\lambda_{j}^{\mathrm{T}_{i}} \rightarrow \infty \quad \text { as } j \rightarrow \infty
$$


Note that $\lambda_{1}^{\mathrm{T}_{i}}=0$ and $v_{1}^{\mathrm{T}_{i}}$ is a constant. Furthermore, the series $\left\{v_{j}^{\mathrm{T}_{i}}\right\}_{j=1}^{\infty}$ forms a complete orthonormal bases in $L^{2}\left(\partial \omega_{i}\right)$. Below we use the notation $(\cdot, \cdot) \partial \omega_{i}$ to denote the inner product on $L^{2}\left(\partial \omega_{i}\right)$. Similarly, we define a local spectral space of dimension $\ell_{i}^{\mathrm{II}}$ and the associated $\ell_{i}^{\mathrm{II}}$-rank projection operator:

$$
\begin{aligned}
V_{\mathrm{off}}^{\mathrm{T}_{i}, \ell_{i}^{\mathrm{II}}} & =\operatorname{span}\left\{v_{j}^{\mathrm{T}_{i}}: 1 \leq j \leq \ell_{i}^{\mathrm{II}}\right\}, \\
\mathcal{P}^{\mathrm{T}_{i}, \ell_{i}^{\ell \mathrm{I}}} v & =\sum_{j=1}^{\ell_{i}^{\mathrm{II}}}\left(v, v_{j}^{\mathrm{T}_{i}}\right)_{\partial \omega_{i}} v_{j}^{\mathrm{T}_{i}} \text { for all } v \in L^{2}\left(\partial \omega_{i}\right) .
\end{aligned}
$$

In addition to these local spectral basis functions defined in Problems (3.2) and (3.11), we need one more local basis function defined by the following local problem:

$$
\left\{\begin{aligned}
-\nabla \cdot\left(\kappa \nabla v^{i}\right) & =\frac{\widetilde{\kappa}}{\int_{\omega_{i}} \widetilde{\kappa} \mathrm{d} x} & & \text { in } \omega_{i}, \\
-\kappa \frac{\partial v^{i}}{\partial n} & =\left|\partial \omega_{i}\right|^{-1} & & \text { on } \partial \omega_{i} .
\end{aligned}\right.
$$

Note that the approximation property of $V_{\text {off }}^{\mathrm{S}_{i}, \ell_{i}^{\mathrm{I}}}, V_{\text {off }}^{\mathrm{T}_{i}, \ell_{i}^{\mathrm{II}}}$ to the local solution $\left.u\right|_{\omega_{i}}$ is of great importance to the analysis of multiscale methods [26, 14]. We present relevant results in Section 4.1]below.

\section{Local harmonic extension bases}

This type of local multiscale bases is defined by local solvers over $\omega_{i}$. The number of such local solvers is problem-dependent. It can be the space of all fine-scale finite element basis functions or the solutions of some local problems with suitable choices of boundary conditions. In this work, we consider the following $\kappa$-harmonic extensions to form the local multiscale space, which has been extensively used in the literature. Specifically, given a fine-scale piecewise linear function $\delta_{j}^{h}(x)$ defined on the boundary $\partial \omega_{i}$, let $\phi_{j}^{\mathrm{H}_{i}}$ be the solution to the following Dirichlet boundary value problem:

$$
\begin{aligned}
-\nabla \cdot\left(\kappa(x) \nabla \phi_{j}^{\mathrm{H}_{i}}\right) & =0 \quad \text { in } \omega_{i}, \\
\phi_{j}^{\mathrm{H}_{i}} & =\delta_{j}^{h} \quad \text { on } \partial \omega_{i},
\end{aligned}
$$

where $\delta_{j}^{h}(x):=\delta_{j, k}$ for all $j, k \in \boldsymbol{J}_{h}\left(\omega_{i}\right)$ with $\delta_{j, k}$ denoting the Kronecker delta symbol, and $\boldsymbol{J}_{h}\left(\omega_{i}\right)$ denoting the set of all fine-grid boundary nodes on $\partial \omega_{i}$. Let $L_{i}$ be the number of the local multiscale functions on $\omega_{i}$. Then the local multiscale space $V_{\text {snap }}^{\mathrm{H}_{i}}$ on $\omega_{i}$ is defined by

$$
V_{\text {snap }}^{\mathrm{H}_{i}}:=\operatorname{span}\left\{\phi_{j}^{\mathrm{H}_{i}}: \quad 1 \leq j \leq L_{i}\right\} .
$$

Its approximation property will be discussed in Section 4.2

\section{Discrete POD}

One challenge associated with the local multiscale space $V_{\text {snap }}^{\mathrm{H}_{i}}$ lies in the fact that its dimensionality can be very large, i.e., $L_{i} \gg 1$, when the problem becomes increasingly complicated in the sense that there are more multiple scales in the coefficient $\kappa$. Thus, the discrete POD is often employed on $\omega_{i}$ to reduce the dimensionality of $V_{\text {snap }}^{\mathrm{H}_{i}}$, while maintaining a certain accuracy. 
The discrete POD proceeds as follows. After obtaining a large number of local multiscale functions $\left\{\phi_{j}^{\mathrm{H}_{i}}\right\}_{j=1}^{L_{i}}$, with $L_{i} \gg 1$, by solving the local problem 3.14), we generate a problem adapted subset of much smaller size from these basis functions by means of singular value decomposition, by taking only left singular vectors corresponding to the largest singular values. The resulting low-dimensional linear subspace with $\ell_{i}$ singular vectors is termed as the offline space of rank $\ell_{i}$.

The auxiliary spectral problem in the construction is to find $\left(\lambda_{j}^{\mathrm{H}_{i}}, v_{j}\right) \in \mathbb{R} \times \mathbb{R}^{L_{i}}$ for $1 \leq j \leq L_{i}$ with the eigenvalues $\left\{\lambda_{j}^{\mathrm{H}_{i}}\right\}_{j=1}^{L_{i}}$ in a nondecreasing order (with multiplicity counted) such that

$$
\begin{aligned}
A^{\text {off }} v_{j} & =\lambda_{j}^{\mathrm{H}_{i}} S^{\text {off }} v_{j}, \\
\left(S^{\text {off }} v_{j}, v_{j}\right)_{\ell^{2}} & =1 .
\end{aligned}
$$

The matrices $A^{\text {off }}, S^{\text {off }} \in \mathbb{R}^{L_{i} \times L_{i}}$ are respectively defined by

$$
A^{\text {off }}=\left[a_{m n}^{\text {off }}\right]=\int_{\omega_{i}} \kappa \nabla \phi_{m}^{\mathrm{H}_{i}} \cdot \nabla \phi_{n}^{\mathrm{H}_{i}} \mathrm{~d} x \quad \text { and } \quad S^{\text {off }}=\left[s_{m n}^{\text {off }}\right]=\int_{\omega_{i}} \widetilde{\kappa} \phi_{m}^{\mathrm{H}_{i}} \cdot \phi_{n}^{\mathrm{H}_{i}} \mathrm{~d} x .
$$

Let $\mathbb{N}_{+} \ni \ell_{i} \leq L_{i}$ be a truncation number. Then we define the discrete POD-basis of rank $\ell_{i}$ by

$$
v_{j}^{\mathrm{H}_{i}}:=\sum_{k=1}^{L_{i}}\left(v_{j}\right)_{k} \phi_{k}^{\mathrm{H}_{i}} \quad \text { for } j=1, \cdots, \ell_{i},
$$

with $\left(v_{j}\right)_{k}$ being the $k^{\text {th }}$ component of the eigenvector $v_{j} \in \mathbb{R}^{L_{i}}$. By the definition of the discrete eigenvalue problem (3.16), we have

$$
\left(v_{j}^{\mathrm{H}_{i}}, v_{k}^{\mathrm{H}_{i}}\right)_{i}=\delta_{j k} \quad \text { and } \quad \int_{\omega_{i}} \kappa \nabla v_{j}^{\mathrm{H}_{i}} \cdot \nabla v_{k}^{\mathrm{H}_{i}} \mathrm{~d} x=\lambda_{j}^{\mathrm{H}_{i}} \delta_{j k} \quad \text { for all } 1 \leq j, k \leq \ell_{i} .
$$

The local offline space $V_{\text {off }}^{\mathrm{H}_{i}, \ell_{i}}$ of rank $\ell_{i}$ is spanned by the first $\ell_{i}$ eigenvectors corresponding to the smallest eigenvalues for problem (3.16):

$$
V_{\mathrm{off}}^{\mathrm{H}_{i}, \ell_{i}}:=\operatorname{span}\left\{v_{j}^{\mathrm{H}_{i}}: \quad 1 \leq j \leq \ell_{i}\right\}
$$

Analogously, we can define a rank $\ell_{i}$ projection operator $\mathcal{P}^{\mathrm{S}_{i}, \ell_{i}}: V_{\text {snap }}^{\mathrm{H}_{i}} \rightarrow V_{\text {off }}^{\mathrm{H}_{i}, \ell_{i}}$ for all $\mathbb{N}_{+} \ni \ell_{i} \leq L_{i}$ by

$$
\mathcal{P}^{\mathrm{H}_{i}, \ell_{i}} v=\sum_{j=1}^{\ell_{i}}\left(v, v_{j}^{\mathrm{H}_{i}}\right)_{i} v_{j}^{\mathrm{H}_{i}} \quad \text { for all } v \in V_{\text {snap }}^{\mathrm{H}_{i}} .
$$

This projection is crucial to derive the error estimate for the discrete POD basis. Its approximation property will be discussed in Section 4.3 .

\subsection{Galerkin approximation}

Next we define three types of global multiscale basis functions based on the local multiscale basis functions introduced in Section 3.1 by partition of unity functions subordinated to the set of coarse neighborhoods $\left\{\omega_{i}\right\}_{i=1}^{N}$. This gives rise to three multiscale methods for solving Problem (1.1) that can approximate reasonably the exact solution $u$ (or the fine-scale solution $u_{h}$ ). 
We begin with an initial coarse space $V_{0}^{\text {init }}=\operatorname{span}\left\{\chi_{i}\right\}_{i=1}^{N}$. The functions $\chi_{i}$ are the standard multiscale basis functions on each coarse element $K \in \mathcal{T}^{H}$ defined by

$$
\begin{aligned}
& -\nabla \cdot\left(\kappa(x) \nabla \chi_{i}\right)=0 \quad \text { in } K, \\
& \chi_{i}=g_{i} \quad \text { on } \partial K \text {, }
\end{aligned}
$$

where $g_{i}$ is affine over $\partial K$ with $g_{i}\left(O_{j}\right)=\delta_{i j}$ for all $i, j=1, \cdots, N$. Recall that $\left\{O_{j}\right\}_{j=1}^{N}$ are the set of coarse nodes on $\mathcal{T}^{H}$.

Remark 3.3 (Properties of $\chi_{i}$ ). The definition (3.20) implies that $\operatorname{supp}\left(\chi_{i}\right)=\omega_{i}$. Thus, we have

$$
\chi_{i}=0 \quad \text { on } \partial \omega_{i} .
$$

Furthermore, the maximum principle implies $0 \leq \chi_{i} \leq 1$. Note that under Assumption 2.1] the gradient of the multiscale basis functions $\left\{\chi_{i}\right\}$ are uniformly bounded [23. Corollary 1.3]

$$
\left\|\nabla \chi_{i}\right\|_{L^{\infty}\left(\omega_{i}\right)} \leq C_{0}
$$

where the constant $C_{0}$ depends on $D$, the size and shape of $D_{j}$ for $j=1, \cdots, m$, the space dimension $d$ and the coefficient $\kappa$, but it is independent of the distances between the inclusions $D_{k}$ and $D_{j}$ for $k, j=$ $1, \cdots, m$. It is worth noting that the precise dependence of the constant $C_{0}$ on $\kappa$ is still unknown. However, when the contrast $\Lambda=\infty$, it is known that the constant $C_{0}$ will blow up as two inclusions approach each other, for which the problem reduces to the perfect or insulated conductivity problem [5]. Such extreme cases are beyond the scope of the present work. The constant $C_{0}$ also depends on coarse grid size $H$ with a possible scaling $H^{-1}$.

Since the set of functions $\left\{\chi_{i}\right\}_{i=1}^{N}$ form partition of unity functions subordinated to $\left\{\omega_{i}\right\}_{i=1}^{N}$, we can construct global multiscale basis functions from the local multiscale basis functions discussed in Section 3.1 [26, 14]. Specifically, the global multiscale spaces $V_{\text {off }}^{\mathrm{S}}, V_{\text {snap }}$ and $V_{\text {off }}^{\mathrm{H}}$ are respectively defined by

$$
\begin{aligned}
V_{\text {off }}^{\mathrm{S}} & :=\operatorname{span}\left\{\chi_{i} v_{j}^{\mathrm{S}_{i}}, \chi_{i} v_{k}^{\mathrm{T}_{i}}, \chi_{i} v^{i}: 1 \leq i \leq N, 1 \leq j \leq \ell_{i}^{\mathrm{I}} \text { and } 1 \leq k \leq \ell_{i}^{\mathrm{II}} \text { with } \ell_{i}^{\mathrm{I}}+\ell_{i}^{\mathrm{II}}=\ell_{i}-1\right\}, \\
V_{\text {snap }} & :=\operatorname{span}\left\{\chi_{i} \phi_{j}^{\mathrm{H}_{i}}: 1 \leq i \leq N \text { and } 1 \leq j \leq L_{i}\right\}, \\
V_{\text {off }}^{\mathrm{H}} & :=\operatorname{span}\left\{\chi_{i} v_{j}^{\mathrm{H}_{i}}: 1 \leq i \leq N \text { and } 1 \leq j \leq \ell_{i}\right\} .
\end{aligned}
$$

Accordingly, the Galerkin approximations to Problem (1.1) read respectively: seeking $u_{\text {off }}^{\mathrm{S}} \in V_{\text {off }}^{\mathrm{S}}, u_{\text {snap }} \in$ $V_{\text {snap }}$ and $u_{\text {off }}^{\mathrm{H}} \in V_{\text {off }}^{\mathrm{H}}$, satisfying

$$
\begin{aligned}
a\left(u_{\text {off }}^{\mathrm{S}}, v\right)=(f, v)_{D} & \text { for all } v \in V_{\text {off }}^{\mathrm{S}}, \\
a\left(u_{\text {snap }}, v\right)=(f, v)_{D} & \text { for all } v \in V_{\text {snap }}, \\
a\left(u_{\text {off }}^{\mathrm{H}}, v\right)=(f, v)_{D} & \text { for all } v \in V_{\text {off }}^{\mathrm{H}} .
\end{aligned}
$$

Note that, by its construction, we have the inclusion relation $V_{\text {off }}^{\mathrm{H}} \subset V_{\text {snap }}$ for all $1 \leq \ell_{i} \leq L_{i}$ with $i=$ $1,2, \cdots, N$. Hence, the Gakerkin orthogonality property [7, Corollary 2.5.10] implies

$$
\left|u-u_{\mathrm{off}}^{\mathrm{H}}\right|_{H_{\kappa}^{1}(D)}^{2}=\left|u-u_{\text {snap }}\right|_{H_{\kappa}^{1}(D)}^{2}+\left|u_{\text {snap }}-u_{\mathrm{off}}^{\mathrm{H}}\right|_{H_{\kappa}^{1}(D)}^{2} .
$$

Furthermore, we will prove in Section 4.3 that $u_{\text {off }}^{\mathrm{H}} \rightarrow u_{\text {snap }}$ in $H_{0}^{1}(D)$, and the convergence rate is determined by $\max _{i=1, \cdots, N}\left\{\left(H^{2} \lambda_{\ell_{i}+1}^{\mathrm{H}_{i}}\right)^{-1 / 2}\right\}$.

The main goal of this work is to derive bounds on the errors $\left|u-u_{\text {off }}^{\mathrm{S}}\right|_{H_{\kappa}^{1}(D)},\left|u-u_{\mathrm{snap}}\right|_{H_{\kappa}^{1}(D)}$ and $\left|u-u_{\mathrm{off}}^{\mathrm{H}}\right|_{H_{\kappa}^{1}(D)}$. This will be carried out in Section 4 below. 


\section{Error estimates}

This section is devoted to the energy error estimates for the multiscale approximations. The general strategy is as follows. First, we derive approximation properties to the local solution $\left.u\right|_{\omega_{i}}$, for the local multiscale

spaces $V_{\text {off }}^{\mathrm{S}_{i}, \ell_{i}^{\mathrm{I}}}, V_{\text {off }}^{\mathrm{T}_{i}, \ell_{i}^{\mathrm{II}}}, V_{\text {snap }}^{\mathrm{H}_{i}}$ and $V_{\text {off }}^{\mathrm{H}_{i}, \ell_{i}}$. Then we combine these local estimates together with partition of unity functions to establish the desired global energy error estimates.

\subsection{Spectral bases approximate error}

Note that the solution $u$ satisfies the following equation

$$
\left\{\begin{aligned}
-\nabla \cdot(\kappa \nabla u) & =f & & \text { in } \omega_{i} \\
-\kappa \frac{\partial u}{\partial n} & =-\kappa \frac{\partial u}{\partial n} & & \text { on } \partial \omega_{i}
\end{aligned}\right.
$$

which can be split into three parts, namely

$$
\left.u\right|_{\omega_{i}}=u^{i, \mathrm{I}}+u^{i, \mathrm{II}}+u^{i, \mathrm{III}}
$$

Here, the three components $u^{i, \mathrm{I}}, u^{i, \mathrm{II}}$, and $u^{i, \mathrm{III}}$ are respectively given by

$$
\left\{\begin{aligned}
-\nabla \cdot\left(\kappa \nabla u^{i, \mathrm{I}}\right) & =f-\bar{f}_{i} & & \text { in } \omega_{i} \\
-\kappa \frac{\partial u^{i, \mathrm{I}}}{\partial n} & =0 & & \text { on } \partial \omega_{i},
\end{aligned}\right.
$$

where $\bar{f}_{i}:=\int_{\omega_{i}} f \mathrm{~d} x \times \frac{\widetilde{\kappa}}{\int_{\omega_{i}} \widetilde{\kappa} \mathrm{d} x}$,

$$
\left\{\begin{aligned}
-\nabla \cdot\left(\kappa \nabla u^{i, \mathrm{II}}\right) & =0 & & \text { in } \omega_{i} \\
-\kappa \frac{\partial u^{i, \mathrm{II}}}{\partial n} & =\kappa \frac{\partial u}{\partial n}-f_{\partial \omega_{i}} \kappa \frac{\partial u}{\partial n} & & \text { on } \partial \omega_{i}
\end{aligned}\right.
$$

and

$$
u^{i, \mathrm{III}}=v^{i} \int_{\omega_{i}} f \mathrm{~d} x
$$

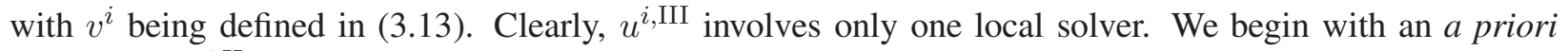
estimate on $u^{i, \mathrm{II}}$.

Lemma 4.1. The following a priori estimate holds:

$$
\left|u^{i, \mathrm{II}}\right|_{H_{\kappa}^{1}\left(\omega_{i}\right)} \leq|u|_{H_{\kappa}^{1}\left(\omega_{i}\right)}+H \mathrm{C}_{\mathrm{poin}}\left(\omega_{i}\right)^{1 / 2}\|f\|_{L_{\kappa-1}^{2}\left(\omega_{i}\right)}
$$

Proof. Let $\widetilde{u}:=u^{i, \mathrm{I}}+u^{i, \mathrm{III}}$. Then it satisfies

$$
\left\{\begin{aligned}
-\nabla \cdot(\kappa \nabla \widetilde{u}) & =f & & \text { in } \omega_{i}, \\
\kappa \frac{\partial \widetilde{u}}{\partial n} & =\frac{1}{\left|\partial \omega_{i}\right|} \int_{\omega_{i}} f \mathrm{~d} x & & \text { on } \partial \omega_{i} .
\end{aligned}\right.
$$


To make the solution unique, we require $\int_{\partial \omega_{i}} \widetilde{u} \mathrm{~d} s=0$. Testing the first equation with $\widetilde{u}$ gives

$$
|\widetilde{u}|_{H_{\kappa}^{1}\left(\omega_{i}\right)}^{2}=\int_{\omega_{i}} f \widetilde{u} \mathrm{~d} x .
$$

Now Poincaré inequality (2.8) and Hölder's inequality lead to

$$
|\widetilde{u}|_{H_{\kappa}^{1}\left(\omega_{i}\right)}^{2} \leq\|f\|_{L_{\kappa^{-1}}^{2}\left(\omega_{i}\right)}\|\widetilde{u}\|_{L_{\kappa}^{2}\left(\omega_{i}\right)} \leq H \mathrm{C}_{\text {poin }}\left(\omega_{i}\right)^{1 / 2}\|f\|_{L_{\kappa^{-1}}^{2}\left(\omega_{i}\right)}|\widetilde{u}|_{H_{\kappa}^{1}\left(\omega_{i}\right)} .
$$

Therefore, we obtain

$$
|\widetilde{u}|_{H_{\kappa}^{1}\left(\omega_{i}\right)} \leq H \mathrm{C}_{\text {poin }}\left(\omega_{i}\right)^{1 / 2}\|f\|_{L_{\kappa^{-1}}^{2}\left(\omega_{i}\right)} \cdot
$$

Finally, the desired result follows from the triangle inequality.

Since $u^{i, \mathrm{I}} \in L_{\widetilde{\kappa}}^{2}\left(\omega_{i}\right), u^{i, \mathrm{II}} \in L^{2}\left(\partial \omega_{i}\right)$, and the series $\left\{v_{j}^{\mathrm{S}_{i}}\right\}_{j=1}^{\infty} \oplus\{1\}$ and $\left\{v_{j}^{\mathrm{T}_{i}}\right\}_{j=1}^{\infty}$ form a complete orthogonal bases in $L_{\widetilde{\kappa}}^{2}\left(\omega_{i}\right)$ and $L^{2}\left(\partial \omega_{i}\right)$, respectively, $u^{i, \mathrm{I}}$ and $u^{i, \text { II }}$ admit the following decompositions:

$$
\begin{aligned}
u^{i, \mathrm{I}} & =c_{0}\left(u^{i, \mathrm{I}}, 1\right)_{i}+\sum_{j=1}^{\infty}\left(u^{i, \mathrm{I}}, v_{j}^{\mathrm{S}_{i}}\right)_{i} v_{j}^{\mathrm{S}_{i}}, \\
u^{i, \mathrm{II}} & =\sum_{j=1}^{\infty}\left(u^{i, \mathrm{II}}, v_{j}^{\mathrm{T}_{i}}\right)_{\partial \omega_{i}} v_{j}^{\mathrm{T}_{i}} .
\end{aligned}
$$

For any $n \in \mathbb{N}_{+}$, we employ the $n$-term truncation $u_{n}^{i, \mathrm{I}}$ and $u_{n}^{i, \mathrm{II}}$ to approximate $u^{i, \mathrm{I}}$ and $u^{i, \mathrm{II}}$, respectively, on $\omega_{i}$ :

$$
u_{n}^{i, \mathrm{I}}:=\mathcal{P}^{\mathrm{S}_{i}, n} u^{i, \mathrm{I}} \in V_{\mathrm{off}}^{\mathrm{S}_{i}, n} \quad \text { and } \quad u_{n}^{i, \mathrm{II}}:=\mathcal{P}^{\mathrm{T}_{i}, n} u^{i, \mathrm{II}} \in V_{\mathrm{off}}^{\mathrm{T}_{i}, n} .
$$

Lemma 4.2. Assume that $f \in L_{\widetilde{\kappa}^{-1}}^{2}(D)$. Then there holds

$$
\left\|f-\bar{f}_{i}\right\|_{L_{\widetilde{\kappa}-1}^{2}\left(\omega_{i}\right)}^{2}=\sum_{j=1}^{\infty}\left(\lambda_{j}^{S_{i}}\right)^{2}\left|\left(u^{i, \mathrm{I}}, v_{j}^{\mathrm{S}_{i}}\right)_{i}\right|^{2}<\infty .
$$

Proof. Since $f \in L_{\widetilde{\kappa}^{-1}}^{2}(D)$, by Lemma $3.2, f-\bar{f}_{i}$ admits the following spectral decomposition:

$$
f-\bar{f}_{i}=\left(\int_{\omega_{i}} \widetilde{\kappa} \mathrm{d} x\right)^{-1}\left(\int_{\omega_{i}}\left(f-\bar{f}_{i}\right) \mathrm{d} x\right) \widetilde{\kappa}+\sum_{j=1}^{\infty}\left(\int_{\omega_{i}}\left(f-\bar{f}_{i}\right) v_{j}^{\mathrm{S}_{i}} \mathrm{~d} x\right) \widetilde{\kappa} v_{j}^{\mathrm{S}_{i}} .
$$

By the definition of $\bar{f}_{i}$, the first term vanishes. Thus, it suffices to compute the $j^{\text {th }}$ expansion coefficient $\int_{\omega_{i}}\left(f-\bar{f}_{i}\right) v_{j}^{\mathrm{S}_{i}} \mathrm{~d} x$ for $j=1,2, \cdots$, which follows from (4.2). Indeed, testing (4.2) with $v_{j}^{\mathrm{S}_{i}}$ yields

$$
\int_{\omega_{i}}\left(f-\bar{f}_{i}\right) v_{j}^{\mathrm{S}_{i}} \mathrm{~d} x=\int_{\omega_{i}} \kappa \nabla u^{i, \mathrm{I}} \cdot \nabla v_{j}^{\mathrm{S}_{i}} \mathrm{~d} x=\lambda_{j}^{\mathrm{S}_{i}} \int_{\omega_{i}} \widetilde{\kappa} u^{i, \mathrm{I}} v_{j}^{\mathrm{S}_{i}} \mathrm{~d} x=\lambda_{j}^{\mathrm{S}_{i}}\left(u^{i, \mathrm{I}}, v_{j}^{\mathrm{S}_{i}}\right)_{i} .
$$

Now we state an important approximation property of the operator $\mathcal{P}^{\mathrm{S}_{i}, \ell_{i}^{\mathrm{I}}}$ of rank $\ell_{i}^{\mathrm{I}}$ defined in 3.10 . 
Proposition 4.1. Assume that $f \in L_{\widetilde{\kappa}^{-1}}^{2}(D)$ and $\ell_{i}^{\mathrm{I}} \in \mathbb{N}_{+}$. Let $u^{i, \mathrm{I}}$ be the first component in 4.1]. Then the projection $\mathcal{P}^{\mathrm{S}_{i}, \ell_{i}^{\mathrm{I}}}: L_{\widetilde{\kappa}}^{2}\left(\omega_{i}\right) \rightarrow V_{\mathrm{off}}^{\mathrm{S}_{i}, \ell_{i}^{\mathrm{I}}}$ of rank $\ell_{i}^{\mathrm{I}}$ defined in (3.10) has the following approximation properties:

$$
\begin{aligned}
\left\|u^{i, \mathrm{I}}-\mathcal{P}^{\mathrm{S}_{i}, \ell_{i}^{\mathrm{I}}} u^{i, \mathrm{I}}\right\|_{L_{\widetilde{\kappa}}^{2}\left(\omega_{i}\right)} & \leq\left(\lambda_{\ell_{i}^{\mathrm{I}}}^{\mathrm{S}_{i}}\right)^{-1}\|f\|_{L_{\widetilde{\kappa}^{-1}}^{2}\left(\omega_{i}\right)}, \\
\left|u^{i, \mathrm{I}}-\mathcal{P}^{\mathrm{S}_{i}, \ell_{i}^{\mathrm{I}}} u^{i, \mathrm{I}}\right|_{H_{\kappa}^{1}\left(\omega_{i}\right)} & \leq\left(\lambda_{\ell_{i}^{\mathrm{I}}}^{\mathrm{S}_{i}}\right)^{-\frac{1}{2}}\|f\|_{L_{\widetilde{\kappa}^{-1}}^{2}\left(\omega_{i}\right)} .
\end{aligned}
$$

Proof. The definitions (4.4) and (3.10), and the orthonormality of $\left\{v_{j}^{S_{i}}\right\}_{j=1}^{\infty} \oplus\{1\}$ in $L_{\widetilde{\kappa}}^{2}\left(\omega_{i}\right)$ directly yield

$$
\begin{aligned}
\left\|u^{i, \mathrm{I}}-\mathcal{P}^{\mathrm{S}_{i}, \ell_{i}^{\mathrm{I}}} u^{i, \mathrm{I}}\right\|_{L_{\tilde{\kappa}}^{2}\left(\omega_{i}\right)}^{2} & =\sum_{j=\ell_{i}^{\mathrm{I}}}^{\infty}\left(u^{i, \mathrm{I}}, v_{j}^{\mathrm{S}_{i}}\right)_{i}^{2}=\sum_{j=\ell_{i}^{\mathrm{I}}}^{\infty}\left(\lambda_{j}^{\mathrm{S}_{i}}\right)^{-2}\left(\lambda_{j}^{\mathrm{S}_{i}}\right)^{2}\left(u^{i, \mathrm{I}}, v_{j}^{\mathrm{S}_{i}}\right)_{i}^{2} \\
& \leq\left(\lambda_{\ell_{i}^{\mathrm{I}}}^{\mathrm{S}_{i}}\right)^{-2} \sum_{j=\ell_{i}^{\mathrm{I}}}^{\infty}\left(\lambda_{j}^{\mathrm{S}_{i}}\right)^{2}\left(u^{i, \mathrm{I}}, v_{j}^{\mathrm{S}_{i}}\right)_{i}^{2} \\
& \leq\left(\lambda_{\ell_{i}^{\mathrm{I}}}^{\mathrm{S}_{i}}\right)^{-2}\left\|f-\bar{f}_{i}\right\|_{L_{\widetilde{\kappa}}^{2}-1}^{2}\left(\omega_{i}\right),
\end{aligned}
$$

where in the last step we have used (4.6). Next, since the first term in the expansion (4.7) vanishes, we deduce that $f-\bar{f}_{i}$ is the $L_{\widetilde{\kappa}^{-1}}^{2}\left(\omega_{i}\right)$ projection onto the codimension one subspace $L_{\widetilde{\kappa}^{-1}}^{2}\left(\omega_{i}\right) \backslash\{\widetilde{\kappa}\}$. Thus,

$$
\left\|f-\bar{f}_{i}\right\|_{L_{\widetilde{\kappa}^{-1}}^{2}\left(\omega_{i}\right)} \leq\|f\|_{L_{\widetilde{\kappa}^{-1}}^{2}\left(\omega_{i}\right)} .
$$

Plugging this inequality into the preceding estimate, we arrive at

$$
\left\|u^{i, \mathrm{I}}-\mathcal{P}^{\mathrm{S}_{i}, \ell_{i}^{\mathrm{I}}} u^{i, \mathrm{I}}\right\|_{L_{\tilde{\kappa}}^{2}\left(\omega_{i}\right)}^{2} \leq\left(\lambda_{\ell_{i}^{\mathrm{I}}}^{\mathrm{S}_{i}}\right)^{-2}\|f\|_{L_{\widetilde{\kappa}-1}^{2}\left(\omega_{i}\right)}^{2}
$$

Taking the square root yields the first estimate. The second estimate can be derived in a similar manner.

Next we give the approximation property of the finite rank operator $\mathcal{P}^{\mathrm{T}_{i}, \ell_{i}^{\mathrm{II}}}$ to the second component of the local solution $u^{i, \mathrm{II}}$, which relies on the regularity of the very weak solution in the appendix.

Lemma 4.3. Let $\ell_{i}^{\mathrm{I}} \in \mathbb{N}_{+}$and let $u^{i, \mathrm{II}}$ be the second component in 4.1). Then the projection $\mathcal{P}^{\mathrm{T}}{ }_{i}, \ell_{i}^{\mathrm{II}}$ : $L^{2}\left(\partial \omega_{i}\right) \rightarrow V_{\mathrm{off}}^{\mathrm{T}_{i}, \ell_{i}}$ of rank $\ell_{i}^{\mathrm{II}}$ defined in (3.12) has the following approximation properties:

$$
\begin{aligned}
&\left\|u^{i, \mathrm{II}}-\mathcal{P}^{\mathrm{T}_{i}, \ell_{i}^{\ell \mathrm{II}}} u^{i, \mathrm{II}}\right\|_{L^{2}\left(\partial \omega_{i}\right)} \leq\left(\lambda_{\ell_{i}^{\mathrm{II}}+1}^{\mathrm{T}_{i}}\right)^{-\frac{1}{2}}\left(|u|_{H_{\kappa}^{1}\left(\omega_{i}\right)}+H \sqrt{\mathrm{C}_{\text {poin }}\left(\omega_{i}\right)}\|f\|_{L_{\kappa^{-1}}^{2}\left(\omega_{i}\right)}\right) \\
&\left\|u^{i, \mathrm{II}}-\mathcal{P}^{\mathrm{T}_{i}, \ell_{i}^{\mathrm{II}}} u^{i, \mathrm{II}}\right\|_{L_{\widetilde{\kappa}}^{2}\left(\omega_{i}\right)} \leq \mathrm{C}_{\text {weak }}\left(\lambda_{\ell_{i}^{\mathrm{II}}+1}^{\mathrm{T}_{i}}\right)^{-\frac{1}{2}}\left(|u|_{H_{\kappa}^{1}\left(\omega_{i}\right)}+H \sqrt{\mathrm{C}_{\text {poin }}\left(\omega_{i}\right)}\|f\|_{L_{\kappa^{-1}}^{2}\left(\omega_{i}\right)}\right), \\
& \int_{\omega_{i}} \chi_{i}^{2} \kappa\left|\nabla\left(u^{i, \mathrm{II}}-\mathcal{P}^{\mathrm{T}_{i}, \ell_{i}^{\mathrm{II}}} u^{i, \mathrm{II}}\right)\right|^{2} \mathrm{~d} x \leq 8 H^{-2} \mathrm{C}_{\text {weak }}^{2}\left(\lambda_{\ell_{i}^{\mathrm{II}}+1}^{\mathrm{T}_{i}}\right)^{-1}\left(|u|_{H_{\kappa}^{1}\left(\omega_{i}\right)}^{2}+H^{2} \mathrm{C}_{\text {poin }}\left(\omega_{i}\right)\|f\|_{L_{\kappa^{-1}}^{2}\left(\omega_{i}\right)}^{2}\right) .
\end{aligned}
$$

Proof. The inequality (4.10) follows from the expansion (4.5), (3.12) and (4.3), and the fact that $u^{i, \mathrm{II}} \in$ $H_{\kappa}^{1}\left(\omega_{i}\right)$. Indeed, we obtain from (4.5) and the orthonomality of $\left\{v_{j}^{\mathrm{T}_{i}}\right\}_{j=1}^{\infty}$ in $L^{2}\left(\partial \omega_{i}\right)$ that

$$
\begin{aligned}
\left\|u^{i, \mathrm{II}}-\mathcal{P}^{\mathrm{T}_{i}, \ell_{i} \mathrm{II}} u^{i, \mathrm{II}}\right\|_{L^{2}\left(\partial \omega_{i}\right)}^{2} & =\sum_{j>\ell_{i}^{\mathrm{II}}}\left|\left(u^{i, \mathrm{II}}, v_{j}^{\mathrm{T}_{i}}\right)_{\partial \omega_{i}}\right|^{2}=\sum_{j>\ell_{i}^{\mathrm{II}}}\left(\lambda_{j}^{\mathrm{T}_{i}}\right)^{-1} \lambda_{j}^{\mathrm{T}_{i}}\left|\left(u^{i, \mathrm{II}}, v_{j}^{\mathrm{T}_{i}}\right) \partial \omega_{i}\right|^{2} \\
& \leq\left(\lambda_{\ell_{i}^{\mathrm{II}}+1}^{\mathrm{T}_{i}}\right)^{-1} \sum_{j>\ell_{i}^{\mathrm{II}}} \lambda_{j}^{\mathrm{T}_{i}}\left|\left(u^{i, \mathrm{II}}, v_{j}^{\mathrm{T}_{i}}\right)_{\partial \omega_{i}}\right|^{2} .
\end{aligned}
$$


Then the estimate (4.10) follows from (4.3) and the identity $\left\langle u^{i, \mathrm{II}}, u^{i, \mathrm{II}}\right\rangle_{i}=\sum_{j=1}^{\infty} \lambda_{j}^{\mathrm{T}_{i}}\left|\left(u^{i, \mathrm{II}}, v_{j}^{\mathrm{T}_{i}}\right)_{\partial \omega_{i}}\right|^{2}$. To prove 4.11, we first write the local error equation for $e:=u^{i, \mathrm{II}}-\mathcal{P}^{\mathrm{T}_{i}, \ell_{i}^{\mathrm{II}}} u^{i, \mathrm{II}}$ by

$$
\left\{\begin{aligned}
-\nabla \cdot(\kappa \nabla e) & =0 & & \text { in } \omega_{i}, \\
e & =u^{i, \mathrm{II}}-\mathcal{P}^{\mathrm{T}_{i}, \ell_{i}^{\mathrm{II}}} u^{i, \mathrm{II}} & & \text { on } \partial \omega_{i} .
\end{aligned}\right.
$$

Now Theorem A.1 yields

$$
\left\|u^{i, \mathrm{II}}-\mathcal{P}^{\mathrm{T}_{i}, \ell_{i}^{\mathrm{II}}} u^{i, \mathrm{II}}\right\|_{L_{\widetilde{\kappa}}^{2}\left(\omega_{i}\right)} \leq \mathrm{C}_{\mathrm{weak}}\left\|u^{i, \mathrm{II}}-\mathcal{P}^{\mathrm{T}_{i}, \ell_{i}^{\mathrm{II}}} u^{i, \mathrm{II}}\right\|_{L^{2}\left(\partial \omega_{i}\right)}
$$

for some constant $\mathrm{C}_{\text {weak }}$ independent of the coefficient $\kappa$. This, together with (4.10), proves (4.11).

To derive the energy error estimate from the $L_{\widetilde{\kappa}}^{2}\left(\omega_{i}\right)$ error estimate, we employ a Cacciopoli type inequality. Note that $\chi_{i}=0$ on the boundary $\partial \omega_{i}$, cf (3.21). Multiplying the first equation in (4.13) with $\chi_{i}^{2} e_{n}$ and then integrating over $\omega_{i}$ and integration by parts lead to

$$
\int_{\omega_{i}} \chi_{i}^{2} \kappa\left|\nabla e_{n}\right|^{2} \mathrm{~d} x=-2 \int_{\omega_{i}} \kappa \nabla e_{n} \cdot \nabla \chi_{i} \chi_{i} e_{n} \mathrm{~d} x .
$$

Together with Hölder's inequality and Young's inequality, we arrive at

$$
\int_{\omega_{i}} \chi_{i}^{2} \kappa\left|\nabla e_{n}\right|^{2} \mathrm{~d} x \leq 4 \int_{\omega_{i}} \kappa\left|\nabla \chi_{i}\right|^{2} e_{n}^{2} \mathrm{~d} x
$$

Further, the definition of $\widetilde{\kappa}$ in 3.3 yields

$$
\int_{\omega_{i}} \chi_{i}^{2} \kappa\left|\nabla e_{n}\right|^{2} \mathrm{~d} x \leq 4 H^{-2} \int_{\omega_{i}} \widetilde{\kappa} e_{n}^{2} \mathrm{~d} x .
$$

Now (4.11) and Young's inequality yield (4.12). This completes the proof of the lemma.

Remark 4.1. It is worth emphasizing that the local energy estimates (4.9) and (4.12) are derived under almost no restrictive assumptions besides the mild condition $f \in L_{\widetilde{\kappa}^{-1}}^{2}(D)$. This estimate is new to the best of our knowledge. The authors [14] utilized the Cacciopoli inequality to derive similar estimates, which, however, incurs some (implicit) assumptions on the problem. Hence, the estimates (4.9) and (4.12) are important for justifying the local spectral approach.

Finally, we present the rank- $\ell_{i}$ approximation to $\left.u\right|_{\omega_{i}}$, where $\ell_{i}:=\ell_{i}^{\mathrm{I}}+\ell_{i}^{\mathrm{II}}+1$ with $\ell_{i}^{\mathrm{I}}, \ell_{i}^{\mathrm{II}} \in \mathbb{N}$ for all $i=1,2, \cdots, N$ :

$$
\widetilde{u}_{i}:=\mathcal{P}^{\mathrm{S}_{i}, \ell_{i}^{\mathrm{I}}} u_{i}^{i, \mathrm{I}}+\mathcal{P}^{\mathrm{T}_{i}, \ell_{i}^{\ell \mathrm{I}}} u_{i}^{i, \mathrm{II}}+u^{i, \mathrm{III}} .
$$

Now, we present an error estimate for the Galerkin approximation $u_{\text {off }}^{\mathrm{S}}$ based on the local spectral basis, cf. (3.24). Our proof is inspired by the partition of unity finite element method (FEM) [26, Theorem 2.1].

Lemma 4.4. Assume that $f \in L_{\widetilde{\kappa}^{-1}}^{2}(D) \cap L_{\kappa^{-1}}^{2}(D)$ and $\ell_{i}^{\mathrm{I}}, \ell_{i}^{\mathrm{II}} \in \mathbb{N}$ for all $i=1,2, \cdots, N$. Let $u$ be the solution to Problem (1.1). Denote $V_{\mathrm{off}}^{\mathrm{S}} \ni w_{\mathrm{off}}^{\mathrm{S}}:=\sum_{i=1}^{N} \chi_{i} \widetilde{u}_{i}$. Then there holds

$$
\begin{aligned}
\left|u-w_{\mathrm{off}}^{\mathrm{S}}\right|_{H_{\kappa}^{1}(D)} & \leq 2 C_{\mathrm{ov}} \max _{i=1, \cdots, N}\left\{\left(H \lambda_{\ell_{i}^{\mathrm{I}}}^{\mathrm{S}_{i}}\right)^{-1}+\left(\lambda_{\ell_{i}^{\mathrm{I}}}^{\mathrm{S}_{i}}\right)^{-\frac{1}{2}}\right\}\|f\|_{L_{\widetilde{\kappa}^{-1}}^{2}(D)} \\
& +7 C_{\mathrm{ov}} \mathrm{C}_{\text {weak }} \mathrm{C}_{\text {poin }} \max _{i=1, \cdots, N}\left\{\left(H^{2} \lambda_{\ell_{i}^{l_{i}^{\mathrm{I}}}+1}^{\mathrm{T}_{1}}\right)^{-\frac{1}{2}}\right\}\|f\|_{L_{\kappa^{-1}}^{2}(D)},
\end{aligned}
$$

where $\mathrm{C}_{\text {poin }}:=\operatorname{diam}(D) \mathrm{C}_{\text {poin }}(D)^{1 / 2}+H \max _{i=1, \cdots, N}\left\{\mathrm{C}_{\text {poin }}\left(\omega_{i}\right)^{1 / 2}\right\}$. 
Proof. Let $e:=u-w_{\text {off }}^{\mathrm{S}}$. Then the property of the partition of unity of $\left\{\chi_{i}\right\}_{i=1}^{N}$ leads to

$$
e=\sum_{i=1}^{N} \chi_{i} e^{i} \quad \text { with } \quad e^{i}:=\left(u_{i}^{\mathrm{I}}-\mathcal{P}^{\mathrm{S}_{i}, \ell_{i}^{\mathrm{I}}} u_{i}^{\mathrm{I}}\right)+\left(u_{i}^{i, \mathrm{II}}-\mathcal{P}^{\mathrm{T}_{i}, \ell_{i}^{\mathrm{II}}} u_{i}^{i, \mathrm{II}}\right):=e_{\mathrm{I}}^{i}+e_{\mathrm{II}}^{i} .
$$

Taking its squared energy norm and using the overlap condition 2.5], we arrive at

$$
\int_{D} \kappa|\nabla e|^{2} \mathrm{~d} x=\int_{D} \kappa\left|\sum_{i=1}^{N} \nabla\left(\chi_{i} e^{i}\right)\right|^{2} \mathrm{~d} x \leq C_{\mathrm{ov}} \sum_{i=1}^{N} \int_{\omega_{i}} \kappa\left|\nabla\left(\chi_{i} e^{i}\right)\right|^{2} \mathrm{~d} x .
$$

This and Young's inequality together imply

$$
\int_{D} \kappa|\nabla e|^{2} \mathrm{~d} x \leq 2 C_{\mathrm{ov}} \sum_{i=1}^{N}\left(\int_{\omega_{i}} \kappa\left|\nabla\left(\chi_{i} e_{\mathrm{I}}^{i}\right)\right|^{2} \mathrm{~d} x+\int_{\omega_{i}} \kappa\left|\nabla\left(\chi_{i} e_{\mathrm{II}}^{i}\right)\right|^{2} \mathrm{~d} x\right) .
$$

It remains to estimate the two integral terms in the bracket. By Cauchy-Schwarz inequality and the definition (3.3) of $\tilde{\kappa}$, we obtain

$$
\begin{aligned}
\int_{\omega_{i}} \kappa\left|\nabla\left(\chi_{i} e_{\mathrm{I}}^{i}\right)\right|^{2} \mathrm{~d} x & \leq 2\left(\int_{\omega_{i}}\left(\kappa \sum_{j=1}^{N}\left|\nabla \chi_{j}\right|^{2}\right)\left|e_{\mathrm{I}}^{i}\right|^{2} \mathrm{~d} x+\int_{\omega_{i}} \kappa \chi_{i}^{2}\left|\nabla e_{\mathrm{I}}^{i}\right|^{2} \mathrm{~d} x\right) \\
& \leq 2\left(H^{-2} \int_{\omega_{i}} \widetilde{\kappa}\left|e_{\mathrm{I}}^{i}\right|^{2} \mathrm{~d} x+\int_{\omega_{i}} \chi_{i}^{2} \kappa\left|\nabla e_{\mathrm{I}}^{i}\right|^{2} \mathrm{~d} x\right) .
\end{aligned}
$$

Then Proposition 4.1 yields

$$
\int_{\omega_{i}} \kappa\left|\nabla\left(\chi_{i} e_{\mathrm{I}}^{i}\right)\right|^{2} \mathrm{~d} x \leq 2\left(\left(H \lambda_{\ell_{i}^{\mathrm{I}}}^{\mathrm{S}_{i}}\right)^{-2}+\left(\lambda_{\ell_{i}^{\mathrm{I}}}^{\mathrm{S}_{i}}\right)^{-1}\right)\|f\|_{L_{\widetilde{\kappa}-1}^{2}\left(\omega_{i}\right)}^{2} .
$$

Analogously, we can derive the following upper bound for the second term:

$$
\int_{\omega_{i}} \kappa\left|\nabla\left(\chi_{i} e_{\mathrm{II}}^{i}\right)\right|^{2} \mathrm{~d} x \leq 20 \mathrm{C}_{\text {weak }}^{2}\left(H^{2} \lambda_{\ell_{i}^{\mathrm{II}}+1}^{\mathrm{T}_{i}}\right)^{-1}\left(|u|_{H_{\kappa}^{1}\left(\omega_{i}\right)}^{2}+H^{2} \mathrm{C}_{\text {poin }}\left(\omega_{i}\right)\|f\|_{L_{\kappa^{-1}}^{2}\left(\omega_{i}\right)}^{2}\right) .
$$

Inserting these two estimate into 4.15) gives

$$
\begin{aligned}
\int_{D} \kappa|\nabla e|^{2} \mathrm{~d} x & \leq 4 C_{\mathrm{ov}} \sum_{i=1}^{N}\left(\left(H \lambda_{\ell_{i}}^{\mathrm{S}_{i}}\right)^{-2}+\left(\lambda_{\ell_{i}^{\mathrm{I}}}^{\mathrm{S}_{i}}\right)^{-1}\right)\|f\|_{L_{\widetilde{\kappa}-1}^{2}}^{2}\left(\omega_{i}\right) \\
& +40 C_{\mathrm{ov}} \sum_{i=1}^{N} \mathrm{C}_{\text {weak }}^{2}\left(H^{2} \lambda_{\ell_{i}^{\mathrm{II}}+1}^{\mathrm{T}_{i}}\right)^{-1}\left(|u|_{H_{\kappa}^{1}\left(\omega_{i}\right)}^{2}+\mathrm{C}_{\text {poin }}\left(\omega_{i}\right) H^{2}\|f\|_{L_{\kappa^{-1}}^{2}\left(\omega_{i}\right)}^{2}\right) .
\end{aligned}
$$

Finally, the overlap condition (2.5) leads to

$$
\begin{aligned}
\int_{D} \kappa|\nabla e|^{2} \mathrm{~d} x & \leq 4 C_{\mathrm{ov}}^{2} \max _{i=1, \cdots, N}\left\{\left(H \lambda_{\ell_{i}^{\mathrm{I}}}^{\mathrm{S}_{i}}\right)^{-2}+\left(\lambda_{\ell_{i}^{\mathrm{I}}}^{\mathrm{S}_{i}}\right)^{-1}\right\}\|f\|_{L_{\kappa^{-1}}^{2}(D)}^{2} \\
& +40 C_{\mathrm{ov}}^{2} \mathrm{C}_{\text {weak }}^{2} \max _{i=1, \cdots, N}\left\{\left(H^{2} \lambda_{\ell_{i}^{\mathrm{II}}+1}^{\mathrm{T}_{i}}\right)^{-1}\right\} \\
& \times\left(|u|_{H_{\kappa}^{1}(D)}^{2}+H^{2} \max _{i=1, \cdots, N}\left\{\mathrm{C}_{\text {poin }}\left(\omega_{i}\right)\right\}\|f\|_{L_{\kappa^{-1}}^{2}(D)}^{2}\right) .
\end{aligned}
$$


Furthermore, since $f \in L_{\kappa^{-1}}^{2}(D)$, we obtain from Poincaré's inequality (2.9) the a priori estimate

$$
|u|_{H_{\kappa}^{1}(D)} \leq \operatorname{diam}(D) \mathrm{C}_{\text {poin }}(D)^{1 / 2}\|f\|_{L_{\kappa^{-1}}^{2}(D)} .
$$

Indeed, we can get by (2.9) that

$$
\int_{D} \kappa u^{2} \mathrm{~d} x \leq \operatorname{diam}(D)^{2} \mathrm{C}_{\text {poin }}(D) \int_{D} \kappa|\nabla u|^{2} \mathrm{~d} x .
$$

Testing (1.1) with $u \in V$, by Hölder's inequality, leads to

$$
\int_{D} \kappa|\nabla u|^{2} \mathrm{~d} x=\int_{D} f u \mathrm{~d} x \leq\|f\|_{L_{\kappa^{-1}}^{2}(D)}\|u\|_{L_{\kappa}^{2}(D)} .
$$

These two inequalities together imply (4.18). Inserting (4.18) into (4.17) shows the desired assertion.

An immediate corollary of Lemma 4.4, after appealing to the Galerkin orthogonality property [7, Corollary 2.5.10], is the following energy error between $u$ and $u_{\mathrm{off}}^{\mathrm{S}}$ :

Proposition 4.2. Assume that $f \in L_{\widetilde{\kappa}^{-1}}^{2}(D) \cap L_{\kappa^{-1}}^{2}(D)$ and let $\ell_{i}^{\mathrm{I}}, \ell_{i}^{\mathrm{II}} \in \mathbb{N}_{+}$for all $i=1,2, \cdots, N$. Let $u \in V$ and $u_{\mathrm{off}}^{\mathrm{S}} \in V_{\mathrm{off}}^{\mathrm{S}}$ be the solutions to Problems (1.1) and (3.24), respectively. There holds

$$
\begin{aligned}
\left|u-u_{\mathrm{off}}^{\mathrm{S}}\right|_{H_{\kappa}^{1}(D)} & :=\min _{w \in V_{\text {off }}^{\mathrm{S}}}|u-w|_{H_{\kappa}^{1}(D)} \\
& \leq \sqrt{2} C_{\mathrm{ov}} \max _{i=1, \cdots, N}\left\{\left(H \lambda_{\ell_{i}^{\mathrm{I}}}^{\mathrm{S}_{i}}\right)^{-1}+\left(\lambda_{\ell_{i}^{\mathrm{I}}}^{\mathrm{S}_{i}}\right)^{-\frac{1}{2}}\right\}\|f\|_{L_{\widetilde{\kappa}-1}^{2}(D)} \\
& +7 C_{\text {ov }} \mathrm{C}_{\text {weak }} \mathrm{C}_{\text {poin }} \max _{i=1, \cdots, N}\left\{\left(H^{2} \lambda_{\ell_{i}^{\mathrm{II}}+1}^{\mathrm{T}_{i}}\right)^{-\frac{1}{2}}\right\}\|f\|_{L_{\kappa^{-1}}^{2}(D)} .
\end{aligned}
$$

Remark 4.2. According to Proposition 4.2 the convergence rate is essentially determined by two factors: the smallest eigenvalue $\lambda_{\ell_{i}}^{\mathrm{S}_{i}}$ that is not included in the local spectral basis and the coarse mesh size $H$. A proper balance between them is necessary for the convergence. For any fixed $H>0$, in view of the eigenvalue problems (3.2) and (3.11), a simple scaling argument implies

$$
H^{2} \lambda_{\ell_{i}^{\mathrm{I}}}^{\mathrm{S}_{i}} \rightarrow \infty \quad \text { and } \quad H \lambda_{\ell_{i}^{\mathrm{II}}}^{\mathrm{T}_{i}} \rightarrow \infty, \quad \text { as } \quad \ell_{i}^{\mathrm{I}}, \ell_{i}^{\mathrm{II}} \rightarrow \infty
$$

Hence, assuming that $\ell_{i}^{\mathrm{I}}$ and $\ell_{i}^{\mathrm{II}}$ are sufficiently large such that $H^{2} \lambda_{\ell_{i}^{\mathrm{II}}}^{\mathrm{S}_{i}} \geq 1$ and $H \lambda_{\ell_{i}^{\mathrm{II}}}^{\mathrm{T}_{i}} \geq H^{-3}$, from Proposition 4.2 we obtain

$$
\left|u-u_{\mathrm{off}}^{\mathrm{S}}\right|_{H_{\kappa}^{1}(D)} \lesssim H\left(\|f\|_{L_{\widetilde{\kappa}^{-1}}^{2}(D)}+\|f\|_{L_{\kappa^{-1}}^{2}(D)}\right)
$$

Note that the estimate of type (4.20) is the main goal of the convergence analysis for many multiscale methods [19] 6] 22]. In practice, the numbers $\ell_{i}^{\mathrm{I}}$ and $\ell_{i}^{\mathrm{II}}$ of local multiscale functions fully determine the computational complexity of the multiscale solver for Problem (3.24) at the offline stage. However, its optimal choice rests on the decay rate of the nonincreasing sequences $\left\{\left(\lambda_{n}^{S_{i}}\right)^{-1}\right\}_{n=1}^{\infty}$ and $\left\{\left(\lambda_{n}^{\mathrm{T}_{i}}\right)^{-1}\right\}_{n=1}^{\infty}$. The precise characterization of eigenvalue decay estimates for heterogeneous problems seems poorly understood at present, and the topic is beyond the scope of the present work. 


\subsection{Harmonic extension bases approximation error}

By the definition of the local harmonic extension snapshot space $V_{\text {snap }}^{\mathrm{H}_{i}}$ in (3.14) and (3.15), there exists $u_{\text {snap }}^{i} \in V_{\text {snap }}^{\mathrm{H}_{i}}$ satisfying

$$
u_{\text {snap }}^{i}:=u_{h} \quad \text { on } \quad \partial \omega_{i} .
$$

In the error analysis below, the weighted Friedrichs (or Poincaré) inequalities play an important role. These inequalities require certain conditions on the coefficient $\kappa$ and domain $D$ that in general are not fully understood. Assumption 2.1 is one sufficient condition for the weighted Friedrichs inequality [16, 27].

Now we can derive the following local energy error estimate.

Lemma 4.5. Let $e_{\text {snap }}^{i}=u_{h}-u_{\text {snap. }}^{i}$. Then there holds

$$
\left|e_{\text {snap }}^{i}\right|_{H_{\kappa}^{1}\left(\omega_{i}\right)} \leq H \sqrt{\mathrm{C}_{\text {poin }}\left(\omega_{i}\right)}\|f\|_{L_{\kappa^{-1}}^{2}\left(\omega_{i}\right)} .
$$

Proof. Indeed, by definition, the following error equation holds:

$$
\left\{\begin{array}{rlrl}
-\nabla \cdot\left(\kappa \nabla e_{\text {snap }}^{i}\right) & =f & & \text { in } \omega_{i} \\
e_{\text {snap }}^{i}=0 & & \text { on } \partial \omega_{i} .
\end{array}\right.
$$

Then (2.8) and Hölder's inequality give the assertion.

Lemma 4.6. Assume that $f \in L_{\kappa^{-1}}^{2}(D)$ and $\ell_{i} \in \mathbb{N}_{+}$for all $i=1,2, \cdots, N$. Let $u_{h} \in V_{h}$ be the unique solution to Problem (2.2). Denote $V_{\text {snap }} \ni w_{\text {snap }}:=\sum_{i=1}^{N} \chi_{i} u_{\text {snap }}^{i}$. Then there holds

$$
\left|u_{h}-w_{\text {snap }}\right|_{H_{\kappa}^{1}(D)} \leq \sqrt{2 C_{\mathrm{ov}}} H \max _{i=1, \cdots, N}\left\{C_{0} H \mathrm{C}_{\text {poin }}\left(\omega_{i}\right)+\sqrt{\mathrm{C}_{\text {poin }}\left(\omega_{i}\right)}\right\}\|f\|_{L_{\kappa}^{2}-1}(D) .
$$

Proof. Let $e_{\text {snap }}:=u_{h}-w_{\text {snap. Since }}\left\{\chi_{i}\right\}_{i=1}^{N}$ forms a set of partition of unity functions subordinated to the set $\left\{\omega_{i}\right\}_{i=1}^{N}$, we deduce

$$
e_{\text {snap }}=\sum_{i=1}^{N} \chi_{i} e_{\text {snap }}^{i}
$$

where $e_{\text {snap }}^{i}:=u_{h}-u_{\text {snap }}^{i}$ is the local error on $\omega_{i}$. Taking its squared energy norm and using the overlap condition 2.5), we arrive at

$$
\int_{D} \kappa\left|\nabla e_{\text {snap }}\right|^{2} \mathrm{~d} x=\int_{D} \kappa\left|\sum_{i=1}^{N} \nabla\left(\chi_{i} e_{\text {snap }}^{i}\right)\right|^{2} \mathrm{~d} x \leq C_{\text {ov }} \sum_{i=1}^{N} \int_{\omega_{i}} \kappa\left|\nabla\left(\chi_{i} e_{\text {snap }}^{i}\right)\right|^{2} \mathrm{~d} x .
$$

It remains to estimate the integral term. Young's inequality gives

$$
\int_{\omega_{i}} \kappa\left|\nabla\left(\chi_{i} e_{\text {snap }}^{i}\right)\right|^{2} \mathrm{~d} x \leq 2\left(\int_{\omega_{i}}\left(\kappa\left|\nabla \chi_{i}\right|^{2}\right)\left|e_{\text {snap }}^{i}\right|^{2} \mathrm{~d} x+\int_{\omega_{i}} \kappa\left|\nabla e_{\text {snap }}^{i}\right|^{2} \mathrm{~d} x\right) .
$$

Taking (3.22) and (2.8) into account, we get

$$
\sum_{i=1}^{N} \int_{\omega_{i}} \kappa\left|\nabla\left(\chi_{i} e_{\text {snap }}^{i}\right)\right|^{2} \mathrm{~d} x \leq 2 \sum_{i=1}^{N}\left(C_{0}^{2} H^{2} \mathrm{C}_{\text {poin }}\left(\omega_{i}\right)+1\right) \int_{\omega_{i}} \kappa\left|\nabla e_{\text {snap }}^{i}\right|^{2} \mathrm{~d} x .
$$


This and 4.22) yield

$$
\sum_{i=1}^{N} \int_{\omega_{i}} \kappa\left|\nabla\left(\chi_{i} e_{\text {snap }}^{i}\right)\right|^{2} \mathrm{~d} x \leq 2 \sum_{i=1}^{N}\left(C_{0}^{2} H^{2} \mathrm{C}_{\text {poin }}\left(\omega_{i}\right)+1\right) \times H^{2} \mathrm{C}_{\text {poin }}\left(\omega_{i}\right)\|f\|_{L_{\kappa^{-1}}^{2}}^{2}\left(\omega_{i}\right) .
$$

Finally, the overlap condition (2.5) and inequality (4.24) show the desired assertion.

Finally, we derive an energy error estimate for the conforming Galerkin approximation to Problem (1.1) based on the multiscale space $V_{\text {snap }}$.

Proposition 4.3. Assume that $f \in L_{\kappa^{-1}}^{2}(D)$. Let $u \in V$ and $u_{\text {snap }} \in V_{\text {snap }}$ be the solutions to Problems (1.1) and (3.25), respectively. Then there holds

$$
\left|u-u_{\text {snap }}\right|_{H_{\kappa}^{1}(D)} \leq \sqrt{2 C_{\mathrm{ov}}} H \max _{i=1, \cdots, N}\left\{C_{0} H \mathrm{C}_{\text {poin }}\left(\omega_{i}\right)+\sqrt{\mathrm{C}_{\text {poin }}\left(\omega_{i}\right)}\right\}\|f\|_{L_{\kappa}^{2}-1}(D)+\min _{v_{h} \in V_{h}}\left|u-v_{h}\right|_{H_{\kappa}^{1}(D)} .
$$

Proof. This assertion follows directly from the Galerkin orthogonality property [7, Corollary 2.5.10], the triangle inequality and the fine-scale a priori estimate (2.3).

\subsection{Discrete POD approximation error}

Now we turn to the discrete POD approximation. First, we present an a priori estimate for Problem (2.2). It will be used to derive the energy estimate for $u_{\text {snap }}^{i}$ defined in 4.21.

Lemma 4.7. Assume that $f \in L_{\kappa^{-1}}^{2}(D)$. Let $u_{h} \in V_{h}$ be the solution to Problem (2.2). Then there holds

$$
\left|u_{h}\right|_{H_{\kappa}^{1}(D)} \leq 2 \operatorname{diam}(D) \sqrt{\mathrm{C}_{\text {poin }}(D)}\|f\|_{L_{\kappa^{-1}}^{2}(D)} .
$$

Proof. In analogy to (4.18), we obtain

$$
\begin{aligned}
|u|_{H_{\kappa}^{1}(D)} & \leq \operatorname{diam}(D) \sqrt{\mathrm{C}_{\text {poin }}(D)}\|f\|_{L_{\kappa^{-1}}^{2}(D)}, \\
\left|u_{h}\right|_{H_{\kappa}^{1}(D)} & \leq \operatorname{diam}(D) \sqrt{\mathrm{C}_{\text {poin }}(D)}\|f\|_{L_{\kappa^{-1}}^{2}(D)} .
\end{aligned}
$$

This and the triangle inequality lead to the desired assertion.

Let $u_{\text {snap }}^{i} \in V_{\text {snap }}^{\mathrm{H}_{i}}$ be defined in (4.21). Then we deduce from (4.22) and the triangle inequality that

$$
\left|u_{\text {snap }}^{i}\right|_{H_{\kappa}^{1}\left(\omega_{i}\right)} \leq H \mathrm{C}_{\text {poin }}\left(\omega_{i}\right)^{1 / 2}\|f\|_{L_{\kappa^{-1}}^{2}\left(\omega_{i}\right)}+\left|u_{h}\right|_{H_{\kappa}^{1}\left(\omega_{i}\right)} .
$$

Note that the series $\left\{v_{j}^{\mathrm{H}_{i}}\right\}_{j=1}^{L_{i}}$ forms a set of orthogonal basis in $V_{\text {snap }}^{\mathrm{H}_{i}}$, cf. (3.18). Therefore, the function $u_{\text {snap }}^{i} \in V_{\text {snap }}^{\mathrm{H}_{i}}$ admits the following expansion

$$
u_{\text {snap }}^{i}=\sum_{j=1}^{L_{i}}\left(u_{\text {snap }}^{i}, v_{j}^{\mathrm{H}_{i}}\right)_{i} v_{j}^{\mathrm{H}_{i}} .
$$


To approximate $u_{\text {snap }}^{i}$ in the space $V_{\text {off }}^{\mathrm{H}_{i}, n}$ of dimension $n$ for some $\mathbb{N}_{+} \ni n \leq L_{i}$, we take its first $n$-term truncation:

$$
u_{n}^{i}:=\mathcal{P}^{\mathrm{H}_{i}, n} u_{\text {snap }}^{i}=\sum_{j=1}^{n}\left(u_{\text {snap }}^{i}, v_{j}^{\mathrm{H}_{i}}\right)_{i} v_{j}^{\mathrm{H}_{i}},
$$

where the projection operator $\mathcal{P}^{\mathrm{H}_{i}, n}$ is defined in 3.19).

The next result provides the approximation property of $u_{n}^{i}$ to $u_{\text {snap }}^{i}$ in the $L_{\widetilde{\kappa}}^{2}\left(\omega_{i}\right)$ norm:

Lemma 4.8. Assume that $f \in L_{\kappa^{-1}}^{2}(D)$. Let $u_{\text {snap }}^{i} \in V_{\text {snap }}^{\mathrm{H}_{i}}$ and $u_{n}^{i} \in V_{\mathrm{off}}^{\mathrm{H}_{i}, n}$ be defined in (4.21) and (4.28) for $\mathbb{N}_{+} \ni n \leq L_{i}$, respectively. Then there holds

$$
\left\|u_{\text {snap }}^{i}-u_{n}^{i}\right\|_{L_{\widetilde{\kappa}}^{2}\left(\omega_{i}\right)} \leq \sqrt{2}\left(\lambda_{n+1}^{\mathrm{H}_{i}}\right)^{-1 / 2}\left(H \sqrt{\mathrm{C}_{\text {poin }}\left(\omega_{i}\right)}\|f\|_{L_{\kappa^{-1}}^{2}\left(\omega_{i}\right)}+\left|u_{h}\right|_{H_{\kappa}^{1}\left(\omega_{i}\right)}\right) .
$$

Proof. It follows from the expansion (4.27) and (3.18) that

$$
\int_{\omega_{i}} \kappa\left|\nabla u_{\text {snap }}^{i}\right|^{2} \mathrm{~d} x=\sum_{j=1}^{L_{i}}\left|\left(u_{\text {snap }}^{i}, v_{j}^{\mathrm{H}_{i}}\right)_{i}\right|^{2} \lambda_{j}^{\mathrm{H}_{i}}
$$

Together with 4.26), we get

$$
\sum_{j=1}^{L_{i}}\left|\left(u_{\text {snap }}^{i}, v_{j}^{\mathrm{H}_{i}}\right)_{i}\right|^{2} \lambda_{j}^{\mathrm{H}_{i}} \leq 2\left(H^{2} \mathrm{C}_{\text {poin }}\left(\omega_{i}\right)\|f\|_{L_{\widetilde{\kappa}^{-1}}^{2}\left(\omega_{i}\right)}^{2}+\left|u_{h}\right|_{H_{\kappa}^{1}\left(\omega_{i}\right)}^{2}\right) .
$$

Meanwhile, the combination of 4.28, (4.27) and (3.18) leads to

$$
\begin{aligned}
\left\|u_{\text {snap }}^{i}-u_{n}^{i}\right\|_{L_{\tilde{\kappa}}^{2}\left(\omega_{i}\right)}^{2} & =\sum_{j=n+1}^{L_{i}}\left|\left(u_{\text {snap }}^{i}, v_{j}^{\mathrm{H}_{i}}\right)_{i}\right|^{2}=\sum_{j=n+1}^{L_{i}}\left(\lambda_{j}^{\mathrm{H}_{i}}\right)^{-1} \lambda_{j}^{\mathrm{H}_{i}}\left|\left(u_{\text {snap }}^{i}, v_{j}^{\mathrm{H}_{i}}\right)_{i}\right|^{2} \\
& \leq\left(\lambda_{n+1}^{\mathrm{H}_{i}}\right)^{-1} \sum_{j=n+1}^{L_{i}} \lambda_{j}^{\mathrm{H}_{i}}\left|\left(u_{\text {snap }}^{i}, v_{j}^{\mathrm{H}_{i}}\right)_{i}\right|^{2} .
\end{aligned}
$$

Further, an application of 4.29) implies

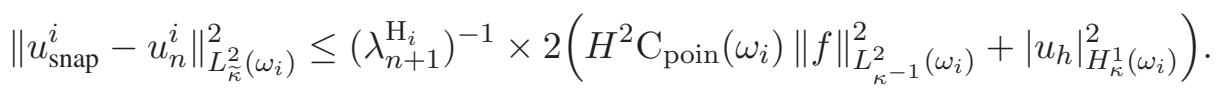

Finally, taking the square root on both sides shows the desired result.

Note that for all $\mathbb{N}_{+} \ni n \leq L_{i}$, both approximations $u_{\text {snap }}^{i}$ and $u_{n}^{i}$ are $\kappa$-harmonic functions. Thus, we can apply the argument in the proof of (4.12) to get the following local energy error estimate.

Lemma 4.9. Let $u_{\text {snap }}^{i} \in V_{\text {snap }}^{\mathrm{H}_{i}}$ and $u_{n}^{i} \in V_{\text {off }}^{\mathrm{H}_{i}, n}$ be defined in (4.21) and (4.28) for all $\mathbb{N}_{+} \ni n \leq L_{i}$. Then there holds

$$
\int_{\omega_{i}} \chi_{i}^{2} \kappa\left|\nabla\left(u_{\text {snap }}^{i}-u_{n}^{i}\right)\right|^{2} \mathrm{~d} x \leq 4 H^{-2} \int_{\omega_{i}} \widetilde{\kappa}\left(u_{\text {snap }}^{i}-u_{n}^{i}\right)^{2} \mathrm{~d} x
$$

Proof. The proof is analogous to that for 4.12), and thus omitted. 
With the help of local estimates presented in Lemmas 4.8 and 4.9 we can now bound the energy error for the POD method by means of the partition of unity FEM [26, Theorem 2.1].

Lemma 4.10. Assume that $f \in L_{\kappa^{-1}}^{2}(D)$. For all $\mathbb{N}_{+} \ni \ell_{i} \leq L_{i}$, denote $V_{\text {snap }} \ni w_{\text {snap }}:=\sum_{i=1}^{N} \chi_{i} u_{\text {snap }}^{i}$ and $V_{\mathrm{off}}^{\mathrm{H}} \ni w_{\mathrm{off}}^{\mathrm{H}}:=\sum_{i=1}^{N} \chi_{i} u_{\ell_{i}}^{i}$. Then there holds

$$
\left|w_{\text {snap }}-w_{\mathrm{off}}^{\mathrm{H}}\right|_{H_{\kappa}^{1}(D)} \leq \sqrt{20 C_{\mathrm{ov}}} \max _{i=1, \cdots, N}\left\{\left(H^{2} \lambda_{\ell_{i}+1}^{\mathrm{H}_{i}}\right)^{-1 / 2}\right\} C_{1}\|f\|_{L_{\kappa}^{2}(D)},
$$

where the constant $C_{1}$ is given by $C_{1}:=H \max _{i=1, \cdots, N}\left\{\sqrt{\mathrm{C}_{\text {poin }}\left(\omega_{i}\right)}\right\}+2 \operatorname{diam}(D) \sqrt{\mathrm{C}_{\text {poin }}(D)}$.

Proof. An argument similar to (4.16) leads to

$$
\left|w_{\text {snap }}-w_{\text {off }}^{\mathrm{H}}\right|_{H_{\kappa}^{1}(D)}^{2} \leq 2 \sum_{i=1}^{N}\left(H^{-2} \int_{\omega_{i}} \widetilde{\kappa}\left|u_{\text {snap }}^{i}-u_{\ell_{i}}^{i}\right|^{2} \mathrm{~d} x+\int_{\omega_{i}} \chi_{i}^{2} \kappa\left|\nabla\left(u_{\text {snap }}^{i}-u_{\ell_{i}}^{i}\right)\right|^{2} \mathrm{~d} x\right) .
$$

Together with Lemma4.9, we obtain

$$
\left|w_{\text {snap }}-w_{\text {off }}^{\mathrm{H}}\right|_{H_{\kappa}^{1}(D)}^{2} \leq 10 H^{-2} \sum_{i=1}^{N} \int_{\omega_{i}} \widetilde{\kappa}\left|u_{\text {snap }}^{i}-u_{\ell_{i}}^{i}\right|^{2} \mathrm{~d} x .
$$

Then from Lemma4.8, we deduce

$$
\left|w_{\text {snap }}-w_{\text {off }}^{\mathrm{H}}\right|_{H_{\kappa}^{1}(D)}^{2} \leq 20 \max _{i=1, \cdots, N}\left\{\left(H^{2} \lambda_{\ell_{i}+1}^{\mathrm{H}_{i}}\right)^{-1}\right\} \sum_{i=1}^{N}\left(H^{2} \mathrm{C}_{\text {poin }}\left(\omega_{i}\right)\|f\|_{L_{\kappa^{-1}}^{2}\left(\omega_{i}\right)}^{2}+\left|u_{h}\right|_{H_{\kappa}^{1}\left(\omega_{i}\right)}^{2}\right) .
$$

Finally, the overlap condition (2.5) together with (4.25) shows the desired assertion.

Finally, we derive an error estimate for the CG approximation to Problem (1.1) based on the discrete POD multiscale space $V_{\text {off }}^{\mathrm{H}}$.

Proposition 4.4. Assume that $f \in L_{\kappa^{-1}}^{2}(D)$ and $\ell_{i} \in \mathbb{N}_{+}$for all $i=1,2, \cdots, N$. Let $u \in V$ and $u_{\mathrm{off}}^{\mathrm{H}} \in V_{\mathrm{off}}^{\mathrm{H}}$ be the solutions to Problems (1.1) and (3.26), respectively. Then there holds

$$
\begin{aligned}
\left|u-u_{\mathrm{off}}^{\mathrm{H}}\right|_{H_{\kappa}^{1}(D)} & \leq \sqrt{2 C_{\mathrm{ov}}} H \max _{i=1, \cdots, N}\left\{C_{0} H \mathrm{C}_{\text {poin }}\left(\omega_{i}\right)+\sqrt{\mathrm{C}_{\text {poin }}\left(\omega_{i}\right)}\right\}\|f\|_{L_{\kappa^{-1}}^{2}(D)} \\
& +\sqrt{20 C_{\mathrm{ov}}} \max _{i=1, \cdots, N}\left\{\left(H^{2} \lambda_{\ell_{i}+1}^{\mathrm{H}_{i}}\right)^{-\frac{1}{2}}\right\} C_{1}\|f\|_{L_{\kappa^{-1}}^{2}(D)}+\min _{v_{h} \in V_{h}}\left|u-v_{h}\right|_{H_{\kappa}^{1}(D)} .
\end{aligned}
$$

Proof. This assertion follows from the Galerkin orthogonality property [7, Corollary 2.5.10], the triangle inequality and the fine-scale a priori estimate (2.3), Proposition 4.3 and Lemma 4.10,

Remark 4.3. Since the discrete eigenvalue problem (3.16) is generated from the continuous eigenvalue problem (3.2) with finite ensembles $\left\{\phi_{j}^{\mathrm{H}_{i}}\right\}_{j=1}^{L_{i}}$, a scaling argument shows

$$
H^{2} \lambda_{n}^{\mathrm{H}_{i}} \rightarrow \infty \quad \text { as } n \rightarrow \infty \text { and } h \rightarrow 0 .
$$

This and (4.30) imply the convergence of the POD solution $u_{\mathrm{off}}^{\mathrm{H}}$ in the energy norm. 


\section{Concluding remarks}

In this paper, we have analyzed three types of multiscale methods in the framework of the generalized multiscale finite element methods (GMsFEMs) for elliptic problems with heterogeneous high-contrast coefficients. Their convergence rates in the energy norm are derived under a very mild assumption on the source term, and are given in terms of the eigenvalues and coarse grid mesh size. It is worth pointing out that the analysis does not rely on any oversampling technique that is typically adopted in existing studies. The analysis indicates that the eigenvalue decay behavior of eigenvalue problems with high-contrast heterogeneous coefficients is crucial for the convergence behavior of the multiscale methods, including the GMsFEM. This motivates further investigations on such eigenvalue problems in order to gain a better mathematical understanding of these methods. Some partial findings along this line have been presented in the work [21], however, much more work remains to be done.

\section{Acknowledgements}

The work was partially supported by the Hausdorff Center for Mathematics, University of Bonn, Germany. The author acknowledges the support from the Royal Society through a Newton international fellowship, and thanks Eric Chung (Chinese University of Hong Kong), Juan Galvis (Universidad Nacional de Colombia, Colombia), Michael Griebel (University of Bonn, Germany) and Daniel Peterseim (University of Augsburg, Germany) for fruitful discussions on the topic of the paper.

\section{A Very-weak solutions to boundary-value problems with high-contrast het- erogeneous coefficients}

In this appendix, we derive a weighted $L^{2}$ estimate for boundary value problems with high-contrast heterogeneous coefficients, which plays a crucial role in the error analysis. Let Assumption 2.1 hold and let $\omega_{i}$ be a coarse neighborhood for any $i=1, \cdots, N$. For any $g \in L^{2}\left(\partial \omega_{i}\right)$, we define the following elliptic problem

$$
\left\{\begin{aligned}
-\nabla \cdot(\kappa \nabla v) & =0 & & \text { in } \omega_{i}, \\
v & =g & & \text { on } \partial \omega_{i} .
\end{aligned}\right.
$$

Our goal is to derive an weighted $L^{2}$ estimate of the solution $v$, which is independent of the high-contrast in the coefficient $\kappa$. To this end, we employ a nonstandard variational form in the spirit of the transposition method [24], and seek $v \in L^{2}\left(\omega_{i}\right)$ such that

$$
-\int_{\omega_{i}} v \nabla \cdot(\kappa \nabla z) \mathrm{d} x=-\int_{\partial \omega_{i}} g \kappa \frac{\partial z}{\partial n} \mathrm{~d} s \quad \text { for all } z \in X\left(\omega_{i}\right) .
$$

Here, $X\left(\omega_{i}\right)$ denotes the test space to be defined below. The main difficulty for our setting of piecewise high-contrast coefficient is that the solution has only piecewise $H^{2}$ regularity, and thus, we cannot directly apply the nonstandard variational form described above. The difficulty is overcome in Theorem A.2.

Theorem A.1. Assume that $\left\{\eta_{j}\right\}_{j=1}^{m}$ are of comparable magnitude and that $\eta_{\min }$ is sufficiently large. Let $g \in L^{2}\left(\omega_{i}\right)$ and let $v$ be the solution to A.1. Then there exists a constant $\mathrm{C}_{\mathrm{weak}}$ independent of the coefficient $\kappa$ such that

$$
\|v\|_{L_{\widetilde{\kappa}}^{2}\left(\omega_{i}\right)} \leq \mathrm{C}_{\text {weak }}\|g\|_{L^{2}\left(\partial \omega_{i}\right)}
$$


To prove it, we need a regularity result based on [8, 21].

Theorem A.2. Assume that $\left\{\eta_{j}\right\}_{j=1}^{m}$ are of comparable magnitude and that $\eta_{\text {min }}$ is sufficiently large. Let $w \in L_{\widetilde{\kappa}^{-1}}^{2}\left(\omega_{i}\right)$ and let $z \in H_{0}^{1}\left(\omega_{i}\right)$ be the unique solution to the following weak formulation

$$
\forall q \in H_{0}^{1}\left(\omega_{i}\right): \int_{\omega_{i}} \kappa \nabla z \cdot \nabla q \mathrm{~d} x=\int_{\omega_{i}} w q \mathrm{~d} x
$$

Then for some constant $\mathrm{C}_{\mathrm{weak}}$ independent of the contrast, there holds

$$
\left\|\eta_{j} \frac{\partial z}{\partial n}\right\|_{L^{2}\left(\partial \omega_{i} \cap D_{j}\right)} \leq \mathrm{C}_{\text {weak }}\|w\|_{L_{\widetilde{\kappa}^{-1}}^{2}\left(\omega_{i}\right)} \quad \text { for all } j=0,1, \cdots, m .
$$

Proof. The triangle inequality, Poincaré inequality, and [21, Eqn. (6.2) and Proposition 6.7] imply

$$
\begin{aligned}
\|z\|_{H^{1}\left(\omega_{i} \cap D_{0}\right)} & \lesssim \mathrm{C}_{\text {poin }}\left(\omega_{i} \cap D_{0}\right)\|w\|_{L^{2}\left(\omega_{i}\right)}, \\
\|z\|_{H^{1}\left(\omega_{i} \cap D_{j}\right)} & \lesssim \eta_{\min }^{-1} \mathrm{C}_{\text {poin }}\left(\omega_{i} \cap D_{0}\right)\|w\|_{L^{2}\left(\omega_{i}\right)}, \quad \text { for } j=1,2, \cdots, m .
\end{aligned}
$$

Note that the $H^{2}$ seminorm regularity result in [8, Theorem B.1] does not depend on the distance between $\partial \omega_{i}$ and $D_{j}$ for any $j=1, \cdots, m$. Therefore, it can be extended to our situation directly:

$$
|z|_{H^{2}\left(\omega_{i} \cap D_{j}\right)} \lesssim \eta_{\min }^{-1}\|w\|_{L^{2}\left(\omega_{i}\right)} \quad \text { for } j=0,1, \cdots, m .
$$

Combining the preceding two estimates and applying interpolation between $H^{1}\left(\omega_{i}\right)$ and $H^{2}\left(\omega_{i}\right)$ yield the $H^{3 / 2}\left(\omega_{i}\right)$ regularity estimate

$$
\|z\|_{H^{3 / 2}\left(\omega_{i} \cap D_{j}\right)} \lesssim \eta_{\min }^{-1}\|w\|_{L^{2}\left(\omega_{i}\right)} .
$$

Furthermore, since $w \in L_{\widetilde{\kappa}^{-1}}^{2}\left(\omega_{i}\right) \subset L^{2}\left(\omega_{i}\right)$, by definition, we can obtain

$$
\begin{aligned}
\|w\|_{L^{2}\left(\omega_{i}\right)}^{2} & =\int_{\omega_{i}} w^{2} \mathrm{~d} x=\sum_{j=0}^{m} \int_{\omega_{i} \cap D_{j}} w^{2} \mathrm{~d} x \\
& \leq \sum_{j=0}^{m} \eta_{j}\|w\|_{L_{\widetilde{\kappa}-1}^{2}\left(\omega_{i} \cap D_{j}\right)}^{2} \lesssim \eta_{\min }\|w\|_{L_{\widetilde{\kappa}-1}^{2}\left(\omega_{i}\right)}^{2} .
\end{aligned}
$$

This, together with A.5), proves

$$
\|z\|_{H^{3 / 2}\left(\omega_{i} \cap D_{j}\right)} \lesssim \eta_{\min }^{-1 / 2}\|w\|_{L_{\widetilde{\kappa}-1}^{2}\left(\omega_{i}\right)}
$$

Since differentiation is continuous from $H^{3 / 2}\left(\omega_{i}\right)$ to $H^{1 / 2}\left(\omega_{i}\right)$, by the trace theorem, we have

$$
\left\|\frac{\partial z}{\partial n}\right\|_{L^{2}\left(\partial \omega_{i} \cap D_{j}\right)} \lesssim\left\|\frac{\partial z}{\partial n}\right\|_{H^{1 / 2}\left(\omega_{i} \cap D_{j}\right)} \lesssim\|z\|_{H^{3 / 2}\left(\omega_{i} \cap D_{j}\right)},
$$

which, together with A.6, proves the desired assertion. 
Next we define a Lions-type variational formulation for Problem (A.1) when $\eta_{\min }$ is large [24, Section 6, Chapter 2]. To this end, let the test space $X\left(\omega_{i}\right) \subset H_{\kappa, 0}^{1}\left(\omega_{i}\right)$ be defined by

$$
X\left(\omega_{i}\right):=\left\{z:-\nabla \cdot(\kappa \nabla z) \in L^{2}\left(\omega_{i}\right) \text { and } z \in H_{\kappa, 0}^{1}\left(\omega_{i}\right)\right\} .
$$

This test space $X\left(\omega_{i}\right)$ is endowed with the norm $\|\cdot\|_{X\left(\omega_{i}\right)}$ :

$$
\forall z \in X\left(\omega_{i}\right):\|z\|_{X\left(\omega_{i}\right)}^{2}=\int_{\omega_{i}} \kappa|\nabla z|^{2} \mathrm{~d} x+\|\nabla \cdot(\kappa \nabla z)\|_{L^{2}\left(\omega_{i}\right)}^{2} .
$$

Below, we denote by $n_{i}(x)$ the unit outward normal (relative to $D_{i}$ ) to the interface $\Gamma_{i}$ at the point $x \in \Gamma_{i}$. For a function $w$ defined on $\mathbb{R}^{2} \backslash \Gamma_{i}$ for $i=1,2, \cdots, m$, we define for any $x \in \Gamma_{i}$,

$$
\left.w(x)\right|_{ \pm}:=\lim _{t \rightarrow 0^{+}} w\left(x \pm t n_{i}(x)\right) \quad \text { and } \quad \frac{\partial}{\partial n_{i}^{ \pm}} w(x):=\lim _{t \rightarrow 0^{+}}\left(\nabla w\left(x \pm t n_{i}(x)\right) \cdot n_{i}(x)\right)
$$

if the limit on the right hand side exists.

Lemma A.1. Let $v$ be the solution to problem a.1 and let the test space $X\left(\omega_{i}\right)$ be defined in A.7. Then the nonstandard variational form (A.2) is well posed.

Proof. For all $z \in X\left(\omega_{i}\right)$, let $w:=-\nabla \cdot(\kappa \nabla z)$, then by definition, $w \in L^{2}\left(\omega_{i}\right)$. Recall the continuity of the flux implied by the definition, i.e.,

$$
\forall z \in X\left(\omega_{i}\right): \quad \eta_{j} \frac{\partial z}{\partial n_{j}^{-}}=\frac{\partial z}{\partial n_{j}^{+}} \quad \text { for all } j=1, \cdots, m .
$$

For all $z \in X\left(\omega_{i}\right)$, we obtain

$$
\begin{aligned}
\int_{\omega_{i}}-\nabla \cdot(\kappa \nabla v) z \mathrm{~d} x & =\sum_{j=0}^{m} \int_{\omega_{i} \cap D_{j}}-\nabla \cdot(\kappa \nabla v) z \mathrm{~d} x=\sum_{j=0}^{m} \int_{\omega_{i} \cap D_{j}}(-\nabla \cdot(\kappa \nabla v z)+\kappa \nabla z \cdot \nabla v) \mathrm{d} x \\
& =\left(\int_{\partial D_{0} \backslash \partial \omega_{i}} \kappa \frac{\partial v}{\partial n_{j}^{+}} z \mathrm{~d} s-\sum_{j=1}^{m} \int_{\partial D_{j} \backslash \partial \omega_{i}} \kappa \frac{\partial v}{\partial n_{j}^{-}} z \mathrm{~d} s\right)+\sum_{j=0}^{m} \int_{\omega_{i} \cap D_{j}} \kappa \nabla z \cdot \nabla v \mathrm{~d} x .
\end{aligned}
$$

The continuity of the flux for $v$ shows that the sum of the first two terms vanishes. We apply the divergence theorem again, together with the continuity of flux for $z$, and derive

$$
\begin{aligned}
\int_{\omega_{i}}-\nabla \cdot(\kappa \nabla v) z \mathrm{~d} x & =\sum_{j=0}^{m} \int_{\omega_{i} \cap D_{j}} \kappa \nabla z \cdot \nabla v \mathrm{~d} x=\sum_{j=0}^{m} \int_{\omega_{i} \cap D_{j}} \nabla \cdot(\kappa \nabla z v)-\nabla \cdot(\kappa \nabla z) v \mathrm{~d} x \\
& =\left(-\int_{\partial D_{0} \backslash \partial \omega_{i}} \kappa \frac{\partial z}{\partial n_{j}^{+}} v \mathrm{~d} s+\sum_{j=1}^{m} \int_{\partial D_{j} \backslash \partial \omega_{i}} \kappa \frac{\partial z}{\partial n_{j}^{-}} v \mathrm{~d} s\right)+\int_{\partial \omega_{i}} \kappa \frac{\partial z}{\partial n} g \mathrm{~d} s \\
& -\int_{\omega_{i}} \nabla \cdot(\kappa \nabla z) v \mathrm{~d} x .
\end{aligned}
$$

The continuity of flux (A.8) indicates that the first term vanishes, and this proves (A.2). 
To prove the well-posedness of the nonstandard variational form A.2 , we introduce a bilinear form $c(\cdot, \cdot)$ on $L_{\widetilde{\kappa}}^{2}\left(\omega_{i}\right) \times L_{\widetilde{\kappa}^{-1}}^{2}\left(\omega_{i}\right)$ and a linear form $b(\cdot)$ on $L_{\widetilde{\kappa}^{-1}}^{2}\left(\omega_{i}\right)$, defined by

$$
\begin{aligned}
c\left(w_{1}, w_{2}\right) & :=\int_{\omega_{i}} w_{1} w_{2} \mathrm{~d} x \quad \text { for all } w_{1} \in L_{\widetilde{\kappa}}^{2}\left(\omega_{i}\right) \text { and } w_{2} \in L_{\widetilde{\kappa}^{-1}}^{2}\left(\omega_{i}\right), \\
b(w) & :=\int_{\partial \omega_{i}} \kappa \frac{\partial z}{\partial n} g \mathrm{~d} s \quad \text { for all } w \in L_{\widetilde{\kappa}^{-1}}^{2}\left(\omega_{i}\right),
\end{aligned}
$$

with $z$ being the unique solution to $\mathrm{A} .3$. It follows from Theorem A.2 that

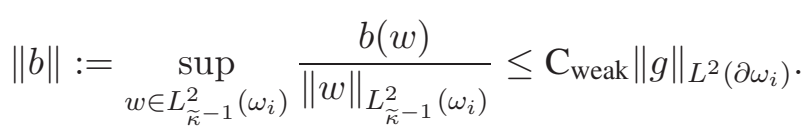

This implies that $b$ lies in the dual space of $L_{\widetilde{\kappa}^{-1}}^{2}\left(\omega_{i}\right)$. Since the dual space of $L_{\widetilde{\kappa}^{-1}}^{2}\left(\omega_{i}\right)$ is $L_{\widetilde{\kappa}}^{2}\left(\omega_{i}\right)$, cf. Remark 3.2 this yields well-posedness of the following variational problem: find $v \in L_{\widetilde{\kappa}}^{2}\left(\omega_{i}\right)$ such that

$$
c(v, w)=b(w) \quad \text { for all } w \in L_{\widetilde{\kappa}^{-1}}^{2}\left(\omega_{i}\right) .
$$

The equivalence of problems (A.10) and A.2 implies the desired well-posedness of A.2).

Finally, we are ready to prove Theorem A.1.

Proof of Theorem A.1 For all $w \in L_{\widetilde{\kappa}^{-1}}^{2}\left(\omega_{i}\right)$, we obtain from (A.9) and A.10)

$$
\int_{\omega_{i}} v w \mathrm{~d} x:=c(v, w)=b(w) \leq \mathrm{C}_{\text {weak }}\|w\|_{L_{\widetilde{\kappa}-1}^{2}\left(\omega_{i}\right)}\|g\|_{\partial \omega_{i}} .
$$

Since $\left(L_{\widetilde{\kappa}^{-1}}^{2}\left(\omega_{i}\right)\right)^{*}=L_{\widetilde{\kappa}}^{2}\left(\omega_{i}\right)$, cf. Remark 3.2 , we get the desired assertion. This completes the proof.

\section{References}

[1] R. Adams and J. Fournier. Sobolev Spaces. Elsevier/Academic Press, Amsterdam, 2003.

[2] G. Alberti, G. Bal, and M. Di Cristo. Critical points for elliptic equations with prescribed boundary conditions. Arch. Ration. Mech. Anal., 226(1):117-141, 2017.

[3] G. Alessandrini and R. Magnanini. Elliptic equations in divergence form, geometric critical points of solutions, and Stekloff eigenfunctions. SIAM J. Math. Anal., 25(5):1259-1268, 1994.

[4] I. Babuska and R. Lipton. Optimal local approximation spaces for generalized finite element methods with application to multiscale problems. Multiscale Model. Simul., 9(1):373-406, 2011.

[5] E. Bao, Y. Li, and B. Yin. Gradient estimates for the perfect and insulated conductivity problems with multiple inclusions. Comm. Partial Differential Equations, 35(11):1982-2006, 2010.

[6] L. Berlyand and H. Owhadi. Flux norm approach to finite dimensional homogenization approximations with non-separated scales and high contrast. Arch. Ration. Mech. Anal., 198(2):677-721, 2010.

[7] S. Brenner and L. Scott. The Mathematical Theory of Finite Element Methods, volume 15 of Texts in Applied Mathematics. Springer, New York, third edition, 2008. 
[8] C.-C. Chu, I. Graham, and T.-Y. Hou. A new multiscale finite element method for high-contrast elliptic interface problems. Math. Comp., 79(272):1915-1955, 2010.

[9] E. Chung, Y. Efendiev, and W. Leung. Generalized multiscale finite element methods for wave propagation in heterogeneous media. Multiscale Model. Simul., 12(4):1691-1721, 2014.

[10] E. Chung, Y. Efendiev, and W. Leung. Constraint energy minimizing generalized multiscale finite element method. Preprint, arXiv:1704.03193, 2017.

[11] W. E and B. Engquist. The heterogeneous multiscale methods. Commun. Math. Sci., 1(1):87-132, 2003.

[12] Y. Efendiev, J. Galvis, and T. Hou. Generalized multiscale finite element methods. J. Comput. Phys., 251:116-135, 2013.

[13] Y. Efendiev, J. Galvis, G. Li, and M. Presho. Generalized multiscale finite element methods. oversampling strategies. Int. J. Multiscale Comput. Eng., 12(6):465-484, 2014.

[14] Y. Efendiev, J. Galvis, and X.-H. Wu. Multiscale finite element methods for high-contrast problems using local spectral basis functions. J. Comput. Phys., 230(4):937-955, 2011.

[15] A. Fraser and R. Schoen. The first Steklov eigenvalue, conformal geometry, and minimal surfaces. Adv. Math., 226(5):4011-4030, 2011.

[16] J. Galvis and Y. Efendiev. Domain decomposition preconditioners for multiscale flows in high-contrast media. Multiscale Model. Simul., 8(4):1461-1483, 2010.

[17] M. Giaquinta. Multiple Integrals in the Calculus of Variations and Nonlinear Elliptic Systems. Princeton University Press, Princeton, NJ, 1983.

[18] T. Hou and X.-H. Wu. A multiscale finite element method for elliptic problems in composite materials and porous media. J. Comput. Phys., 134(1):169-189, 1997.

[19] T. Hughes, G. Feijóo, L. Mazzei, and J.-B. Quincy. The variational multiscale method—a paradigm for computational mechanics. Comput. Methods Appl. Mech. Engrg., 166(1-2):3-24, 1998.

[20] R. Laugesen. Spectral Theory of Partial Differential Equations. Lecture Notes, UIUC. https://wiki.illinois.edu//wiki/display/MATH595STP/Math+595+STP (last accessed on December 20, 2017).

[21] G. Li. Low-rank approximation to heterogeneous elliptic problems. Multiscale Model. Simul., page in press, 2017.

[22] G. Li, D. Peterseim, and M. Schedensack. Error analysis of a variational multiscale stabilization for convection-dominated diffusion equations in two dimensions. IMA J. Numer. Anal., pages in press, https://doi.org/10.1093/imanum/drx027, 2017.

[23] Y. Li and M. Vogelius. Gradient estimates for solutions to divergence form elliptic equations with discontinuous coefficients. Arch. Ration. Mech. Anal., 153(2):91-151, 2000.

[24] J.-L. Lions and E. Magenes. Non-homogeneous Boundary Value Problems and Applications. Vol. I. Springer-Verlag, New York-Heidelberg, 1972. 
[25] A. Målqvist and D. Peterseim. Localization of elliptic multiscale problems. Math. Comp., 83(290):2583-2603, 2014.

[26] J. Melenk and I. Babuška. The partition of unity finite element method: basic theory and applications. Comput. Methods Appl. Mech. Engrg., 139(1-4):289-314, 1996.

[27] C. Pechstein and R. Scheichl. Weighted Poincaré inequalities. IMA J. Numer. Anal., 33(2):652-686, 2012.

[28] K. Yosida. Functional Analysis. Springer-Verlag, Berlin-New York, 1980. 